\title{
Molecular Insight in the Optical Response of Tubular Chlorosomal Assemblies
}

\author{
Xinmeng Li, ${ }^{*}$ Francesco Buda, ${ }^{\circledR}$ Huub J. M. de Groot, and G. J. Agur. Sevink*() \\ Leiden University, Leiden Institute of Chemistry, Einsteinweg 55, P.O. Box 9502, 2300 RA Leiden, The Netherlands
}

Supporting Information

\begin{abstract}
A systematic procedure is developed for determining unique stackings of $\mathrm{BChl}$ molecules in different types of chlorosomes directly from characterization data, using information about: (i) the dimeric unit cell from nuclear magnetic resonance (NMR), (ii) local stacking distances from cryo-electron microscopy (cryo-EM), and (iii) large-scale chirality from absorption, linear-dichroism, and circular-dichroism spectra. To enable a comparison with optical data, we have employed a Frenkel Hamiltonian formalism to calculate optical properties of preassembled tubes of realistic $120 \mathrm{~nm}$ length. Our analysis for the first time explains the variability of optical signals measured for chlorosomes and points at chiral angles $\delta=$

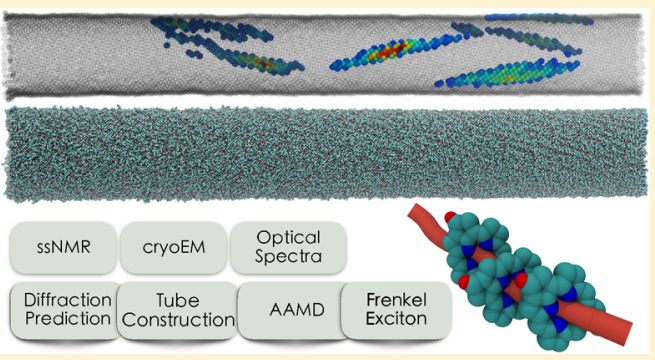
$105^{*}$ for $\mathrm{BChl} c$ and $\delta=19.8^{\circ}$ for BChl $d$ that satisfy all information contained in parts i-iii within experimental and computational accuracy, where the asterisk denotes that the large-scale tube curvature is reversed. We also find that the dynamic disorder is not sensitive to a particular chirality. To investigate the role of disorder on excitonic features, we have represented correlated deviations from a crystalline order due to thermal energy, as extracted from all-atom molecular dynamics (AAMD) calculations, directly in the coupling terms of the Frenkel Hamiltonian, assuming that variations of site energies can be neglected. The use of AAMD snapshot for sampling relevant molecular conformations allows us to study molecular origins of important emergent phenomena in chlorosomes. We find that our model reproduces two mechanisms-exciton localization and level crossing - that have been proposed to play a key role in the transfer of excitonic energy. This highlights the importance of the usually disregarded rotation-related electronic coupling variations in the exciton properties.
\end{abstract}

\section{INTRODUCTION}

Chlorosomes are assemblies of bacteriochlorophylls (BChls) that enable light harvesting at very low light intensities as well as highly efficient transfer of excitonic energy in green sulfur bacteria. ${ }^{1}$ They are intriguing examples of how nature manages to secure efficiency and robustness without protein-based encoding and structural frameworks, rendering an improved understanding of their design principles a primary research target for the development of smart nanodevices for lightharvesting. Nuclear magnetic resonance (NMR) and cryoelectron microscopy (cryo-EM) have contributed to a better understanding of short-range structural properties in chlorosomes of different organisms that each feature a specific composition. ${ }^{2}$ Limited information on the secondary structural organization for any of the studied chlorosomes or their reconstituted analogues and incomplete insight how to resolve their secondary structural based on the available experimental information pose a challenge to a better understanding of chlorosomal function encoding.

The evasiveness of the higher order structural regularity is usually attributed to gross heterogeneity, which encompasses heterogeneity in size, overall shape, and in composition, as well as static and dynamic disorder in the internal organization of molecules on an overall regular lattice. ${ }^{3}$ Nevertheless, the widths of spectroscopic signals for individual wild type (WT) chlorosomes provide strong indications that there is consid- erable regularity in the $\mathrm{BChl}$ positioning even at larger scales and suggest a cylindrical symmetry. ${ }^{4}$ The accepted structural model for chlorosomes is that of a concentric tubular superstructure generated by wrapping curved sheets of stacks of syn-anti parallel dimers. ${ }^{2}$ Within sheets, dimers are thought to be loosely positioned on a $2 \mathrm{D}$ Bravais lattice and held together by an interaction network of $\pi-\pi$ overlap, coordination, and hydrogen bonding. ${ }^{5}$

Combining all-atom molecular dynamics (AAMD) simulations and geometrical analysis, we recently reconsidered the lattice dimensions, and we determined a small set of chiral angles $\delta$ that agree with the cryo-EM derived power spectra upon $2 \mathrm{D}$ lattice-to-cylinder projection. ${ }^{5}$ Moreover, we identified dynamic heterogeneity in terms of a rotational degree of freedom of $\mathrm{BChl}$ molecules, which is closely linked to the formation and breakage of hydrogen-bonded domains. This degree of freedom is the hallmark of a plastic crystal, in which weakly interacting molecules possess orientational or conformational degrees of freedom within a regular, crystalline framework. Such intrinsic structural heterogeneity can only be represented at atomic resolution.

Received: April 26, 2019

Revised: June 7, 2019

Published: June 7, 2019 
The current study has a dual focus. On one hand, we further refine our matching procedure by also including optical data in the matching criteria. The starting point for the comparison of calculated optical spectra is the usual Frenkel Hamiltonian ${ }^{6}$ and preassembled $120 \mathrm{~nm}$ long tubes with chirality $\delta$ determined by our geometrical analysis. Moreover, in contrast to studies that only compare a single spectrum, ${ }^{7}$ we uniquely account for the absorption, linear, and circular dichroism spectra simultaneously in our matching procedure. Our analysis shows that three sources of data-NMR, cryo-EM and optical spectrasuffice to single out chiral angles $\delta$ for both wild-type or mutant chlorosomes. Previously, Günther et al. ${ }^{7}$ also concluded that combined data are required for unambiguous determination of chirality, but they analyzed the angle-dependent absorption spectrum upon excitation in terms of an idealized lattice model. $^{7}$

Next, we have analyzed the excitonic properties of tubular assembles along the AAMD trajectory in order to investigate how disorder at a molecular level relates to the unique excitonic properties of chlorosomes. ${ }^{8}$ We concentrate on both single and three-layered tubes of $\mathrm{BChl} c$. In our Frenkel Hamiltonian treatment, we account for explicit disorder only in the coupling between different molecules, i.e., the off-diagonal terms of the Hamiltonian, based on our previous finding that molecular rotation is an important dynamical feature. Since exciton delocalization relates to only very weak energetic disorder when compared to coupling, ${ }^{9}$ we choose to neglect fluctuations in the diagonal terms. We show that our current treatment is indeed capable of capturing two proposed mechanisms for exciton transfer-localization and level crossing - and thus allows us to rationalize their molecular origins. Our treatment goes well beyond existing computational studies that are based on regular lattices $^{6,10-12}$ or investigate disorder in terms of random perturbations of Hamiltonian elements, which ignore correlations in both the small- and large-scale tube dynamics.

The main achievements of this study are (i) the development of a procedure that maps heterogeneous data to unique $\mathrm{BChl}$ packings at multiple scales; (ii) the first molecular insight into the role of disorder on the properties of superradiant excitonic domains in tubular chlorosomes, extending insight obtained from $2 \mathrm{D}$ spectroscopy measurements; ${ }^{13}$ and (iii) insight into the sensitivity of the excited state to the dynamic disorder, indicating the key role of correlated molecular motion in the EET of chlorosomes. ${ }^{14}$

\section{COMPUTATIONAL METHODS}

2.1. Building and Simulating Molecular Assemblies. For details about our computational approach and unit cell parameters for syn-anti dimers of BChl $c$ and BChl $d$, we refer to our previous study. ${ }^{5}$ All AAMD simulations in vacuo were performed in a canonical ensemble (NVT), using a V-rescale thermostat $^{15}$ and periodic boundary conditions. To avoid interference with periodic mirrors, the tube structures are centered in a large simulation volume of $30 \times 30 \times 140 \mathrm{~nm}^{3}$, with at least $7.5 \mathrm{~nm}$ distance to any boundary. Tubular superstructures with predefined chiral angles are grown through one-by-one addition of dimeric unit cells. The approximate sizes of the resulting optimized tubes are $120 \mathrm{~nm}$ (length) and $15 \mathrm{~nm}$ (diameter), which are close to the actual dimensions in nature. ${ }^{1}$ To alleviate possible molecular frustration and partial overlap of tail parts resulting from the growth algorithm, and to ensure stable AAMD, we first perform standard structure optimization by steepest descent for the complete structure.
Structural disorder in the head part after energy minimization is found to be negligible, thus this structure, labeled $T=0 \mathrm{~K}$, can be seen as ordered. Subsequently, a short (100 ps) MD simulation at $50 \mathrm{~K}$ is carried out with the position of atoms in the head part fixed, in order to further relax the configuration of the (farnesyl) tail part of the molecules. After this step, all constraints are relieved, and a $1 \mathrm{~ns} \mathrm{MD}$ simulation is performed at $50 \mathrm{~K}$ to introduce mild structural disorder. Finally, the temperature is increased to $300 \mathrm{~K}$, and the system is evolved for $1 \mathrm{~ns}$ at the ambient temperature, which is expected to result in stronger structural disorder.

To investigate the sensitivity of the excited states to the dynamic disorder, we generated MD trajectories for single-tube and concentric-tube with two different time resolutions: one dilute sampling with one configuration every $20 \mathrm{ps}$, in total 500 ps, and one dense sampling with one configuration every $20 \mathrm{fs}$, in total 1 ps. Since calculating the optical response is computationally intensive, the tube length for both trajectories is $60 \mathrm{~nm}$ instead of the usual $120 \mathrm{~nm}$. We have confirmed computationally that the spectra features show very little length dependence in the range from 60 to $120 \mathrm{~nm}$ (see Supporting Information).

2.2. Calculating Optical Spectra. We adopt the Frenkel Exciton Hamiltonian ${ }^{6,10}$

$$
\hat{H}=\sum_{i} v_{i}(\mathbf{R})|i\rangle\left\langle i\left|+\sum_{i \neq k} J_{i k}(\mathbf{R})\right| k\right\rangle\langle i| \quad i, k \in[1, N]
$$

to calculate the optical response of chlorosome tube aggregates composed of $N$ molecules. Here, atomistic detail contained in the coordinates $\mathbf{R}$ is explicit in the $\nu_{i}(\mathbf{R})$ on the diagonal, representing the monomer excitation energy of the molecule at site $i$, and in the off-diagonal terms, $J_{i k}(\mathbf{R})$, which represent the electronic coupling between molecules $i$ and $k$. In the next subsection, we discuss the different options for representing this molecular detail. For ordered helical lattice tube models, ${ }^{6,7,10-12}$ i.e., in the homogeneous limit, $\nu_{i}(\mathbf{R})$ and $J_{i k}(\mathbf{R})$ are independent of molecular details and $\nu_{i}(\mathbf{R})=\nu$ is a constant value, while $J_{i k}(\mathbf{R})$ is given by a simple geometrical relation depending on the distance vector $R$. However, when one includes microscopic molecular detail to account for the disorder that is eminent in most photosynthetic structures, ${ }^{16}$ one has to consider the R-dependence explicitly. For the electronic coupling $J_{i k}(\mathbf{R})$, we consider the standard point dipole approximation (PDA)

$$
J_{i k}(\mathbf{R})=\frac{\vec{\mu}_{i} \cdot \vec{\mu}_{k}}{\left|\overrightarrow{\mathrm{R}}_{i k}\right|^{3}}-3 \frac{\left(\vec{\mu}_{i} \cdot \overrightarrow{\mathrm{R}}_{i k}\right)\left(\vec{\mu}_{k} \cdot \overrightarrow{\mathrm{R}}_{i k}\right)}{\left|\overrightarrow{\mathrm{R}}_{i k}\right|^{5}}
$$

with $\vec{\mu}_{i}=\vec{\mu}_{i}(\mathbf{R})$ the effective transition dipole moment vector for molecule $i$ and $\overrightarrow{\mathrm{R}}_{i k}$ the distance vector between the two transition dipoles labeled by $i$ and $k .{ }^{17}$ We note that the PDA stems from the leading term in a multipole expansion of the Coulomb coupling, and is known to overestimate the coupling strengths at distances that are small compared to the spatial dimension of the molecules. ${ }^{18}$ Nevertheless, the PDA is commonly employed for densely packed BChls in chlorosomes instead of the more elaborate methods that derive couplings from quantum-chemical calculations or efficient transition charges from electrostatic potentials (TrEsp) estimates, ${ }^{18}$ because calculated red shifts based on PDA-coupling and empirical parameters were found to consistently match measured values. ${ }^{6,7,10,11,19-21}$ In our case, all off-diagonal coupling terms in the Hamiltonian are calculated using dipoles 
that are extracted from coordinates $\mathbf{R}$ of our AAMD trajectory. For reasons explained in the next subsection, we consider a constant site energy in the diagonal terms of $\hat{H}$. The general form of the density matrix describing the excited state manifold for the system at time $t$ after photoexcitation is

$$
\rho(t)=\sum_{i=1}^{N} \sum_{j=1}^{N} \rho_{i j}(t)|i\rangle\langle j|
$$

where $\rho_{i j}(t)$ describes the populations $(i=j)$ and coherences $(i$ $\neq j)$ in the site basis. ${ }^{9}$ In the present study, we do not consider exciton dynamics explicitly.

We select a site energy $\nu=15390 \mathrm{~cm}^{-1}$ that was derived from the $\mathrm{Q}_{y}$-transition of isolated $\mathrm{BChl} c$ in methanol for the monomer excitation energy. ${ }^{6}$ For simplicity, we assign the same monomer excitation energy $\nu$ to $\mathrm{BChl} d$, as slightly shifted values will only lead to an overall translation of spectra without changing the spectral shapes. The transition dipole moment vector $\vec{\mu}_{i}$ is taken oriented along the vector connecting two nitrogen atoms $\left(N_{\mathrm{I}} \text { and } N_{\mathrm{III}}\right)^{19}$ in agreement with the transition dipole moment determined in a recent theoretical TD-DFT study. ${ }^{22}$ The length $\left|\vec{\mu}_{i}\right|$ of this vector is $5.48 \mathrm{D}$, which is estimated from experimental data ${ }^{23}$ and used in several studies. ${ }^{6,11,19,20}$ We note that an additional factor 5.04 enters $J_{i k}$ due to a conversion of Debye to units of $\mathrm{cm}^{-1} \cdot{ }^{24}$

Diagonalization of the Hamiltonian matrix $\hat{H}$ provides $N$ eigenvectors $\left|\psi_{m}\right\rangle$ and eigenvalues $\varepsilon_{m}$ that correspond to the stationary exciton state and excitonic transition energy, i.e., $\hat{H}\left|\psi_{m}\right\rangle=\varepsilon_{m}\left|\psi_{m}\right\rangle$. As $\hat{H}$ is both real and symmetric, $\varepsilon_{m}$ are real and the wave functions $\left|\psi_{m}\right\rangle$, providing a state basis for the description of the excited system, can also be chosen to be real., ${ }^{61}$ Eigenvectors are usually sorted with respect to the excitonic transition energy, from lowest to highest. A spatial analysis of these state vectors is of vital importance for understanding the excitonic behavior of chlorosomes. For this purpose, a state vector $\left|\psi_{m}\right\rangle$ can be represented on the site basis $|i\rangle$

$$
\left|\psi_{m}\right\rangle=\sum_{i=1}^{N} c_{m i}|i\rangle
$$

with $|i\rangle$ a state where only the molecule at site $i$ is excited, while all other molecules are in the ground state. In the site basis view, the contribution of a specific site $i$ involved in the state $\left|\psi_{m}\right\rangle$ is given by $p_{i}^{m}=\left|\left\langle i \mid \psi_{m}\right\rangle\right|^{2}=\left|c_{m i}\right|^{2}$, which is often called the exciton density of the molecule at site $i$ and can be visualized by color coding. The spatial extent of an excitonic state $\left|\psi_{m}\right\rangle$ is quantified by the inverse participation ratio: ${ }^{1,25,26}$

$$
\mathrm{IPR}_{m}=\frac{1}{\sum_{i=1}^{N}\left|c_{m i}\right|^{4}}
$$

with IPR = 1 for a fully localized state, i.e. confined to a single site, and IPR $=N$ for a state that is fully delocalized over all sites in the tube. In the remainder, we concentrate on the exciton state with the largest oscillator strength, which is often called the absorption state or $\psi_{\max }$ for brevity. ${ }^{11}$

Each exciton state $\psi_{m}$ corresponds to a wavelength equals to the corresponding eigenvalue $\varepsilon_{m}$ (horizontal position) and a signal strength (vertical axis) $d_{m}$ for absorption (OD), $\mathrm{LD}_{m}$ for linear dichroism (LD) and $\mathcal{R}_{m}$ for circular dichroism (CD), which have been calculated according to the known expressions ${ }^{6,27}$

$$
\begin{aligned}
d_{m} & =\sum_{i, k=1}^{N}\left(\vec{\mu}_{i} \cdot \vec{\mu}_{k}\right) c_{m i} c_{m k} \\
\mathrm{LD}_{m} & =d_{m}^{\|}-d_{m}^{\perp}=\frac{1}{2} \sum_{i, k=1}^{N}\left[3\left(\vec{\mu}_{i} \cdot \overrightarrow{\mathrm{e}}\right)\left(\vec{\mu}_{k} \cdot \overrightarrow{\mathrm{e}}\right)-\vec{\mu}_{i} \cdot \vec{\mu}_{k}\right] c_{m i} c_{m k} \\
\mathcal{R}_{m} & =1.7 \times 10^{-5} \sum_{i, k=1}^{N} \varepsilon_{m}\left[\vec{R}_{i k} \cdot\left(\vec{\mu}_{k} \times \vec{\mu}_{i}\right)\right] c_{m i} c_{m k}
\end{aligned}
$$

Here, $\overrightarrow{\mathrm{e}}$ is a unit vector along the long axis of the tube. In order to relate our results to earlier publications, stick spectra calculated using (6) have been convoluted with a normalized Gaussian profile in a postprocessing step. This operation mimics the experimental variability that leads to signal broadening, which stems from both the static and dynamic disorder that is present in the studied chlorosomes. Since several estimates have been previously used, ${ }^{6}$ we show spectra plots for range of fwhm values from 10 to $350 \mathrm{~cm}^{-1}$.

2.3. Parameters in the Frenkel Hamiltonian. A key limitation in using the Frenkel formalism for large-scale assemblies like chlorosomes is the difficulty to determine all terms in the Hamiltonian $\hat{H}$ microscopically using ab initio calculations. Thus, in order to come to a computable $\hat{H}$, several types of approximations have to be made. A common approximation is to account for R-dependent contributions only in diagonal elements of $\hat{H}$, i.e. in structure-dependent variation of the site energy. While this approximation may be warranted for pigment-protein complexes, it ignores the effect of varying electronic coupling $J_{i k}(\mathbf{R})$, i.e., the off-diagonal terms, due to the substantial rotational twisting motions of the BChl head groups that has been observed along the AAMD trajectory. Electronic coupling is seen as a key factor for the fast electronic energy transfer (EET) process in photosynthesis complexes. $^{28}$ We note that, for our simulated structures, the influence of different $\mathrm{BChl}$ homologues in the structure, structural defects and variations, and vibrational modes that are slow compared to the exciton life times, is absent or fairly limited. For this reason, the contribution of static disorder to $\nu_{i}(\mathbf{R})$ is not discussed here.

Several groups have attempted to quantify $\nu_{i}(\mathbf{R})$. The contribution of changing molecular configurations and/or their environment to a dynamic modulation $\Delta \nu_{i}(\mathbf{R})=\nu_{i}(\mathbf{R})$ $-\nu_{i}^{0}$ can in principle be evaluated using combined quantum chemical/electrostatic methods for a fairly limited number of residues. ${ }^{18,29-32}$ However, even in such cases, calculating electronic excited states with proper accuracy remains a challenge. ${ }^{29}$ For example, the accuracy of TD-DFT calculations is limited to $0.5-0.4 \mathrm{eV}, 29$ and using the ground state potential surface sampled by MD for TD-DFT calculations is known to overestimate fluctuations of the excitation energy. ${ }^{32}$ For chlorosomes, the task of calculating $\nu_{i}(\mathbf{R})$ for varying nuclear coordinates $\mathbf{R}$ is computationally intractable even for modern supercomputers, and thus it is always replaced by a more tractable task. The simplest choice is to assume a constant $\nu_{i}^{0}$, which is estimated from experiments, following the argument that the environments of BChls are very similar on average, ${ }^{33}$ and the observation that the HOMO-LUMO gaps in chlorophylls can be quite insensitive to external perturbations, that affect the energy levels, because of correlated instead of anticorrelated variation upon interaction with the environment. ${ }^{34}$ The most notable example of this effect is found in 
Table 1. $\delta \in\left[0^{\circ}, 180^{\circ}\right]$ for BChl $c(\mathrm{WT})$ and BChl $d(b c h Q R U)$ Assemblies That Reproduce Layer Line Positions $1 / d=1.25$ $\mathrm{nm}^{-1}$ and $1 / d=0.83 \mathrm{~nm}^{-1}$ for WT or $b c h Q R U$ chlorosomes ${ }^{a}$

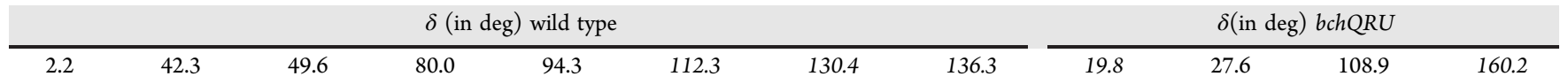

${ }^{a}$ The italicized $\delta$ values in the table indicate that the tube for that $\delta$ displays the experimentally observed meridian signal on the target layer line.
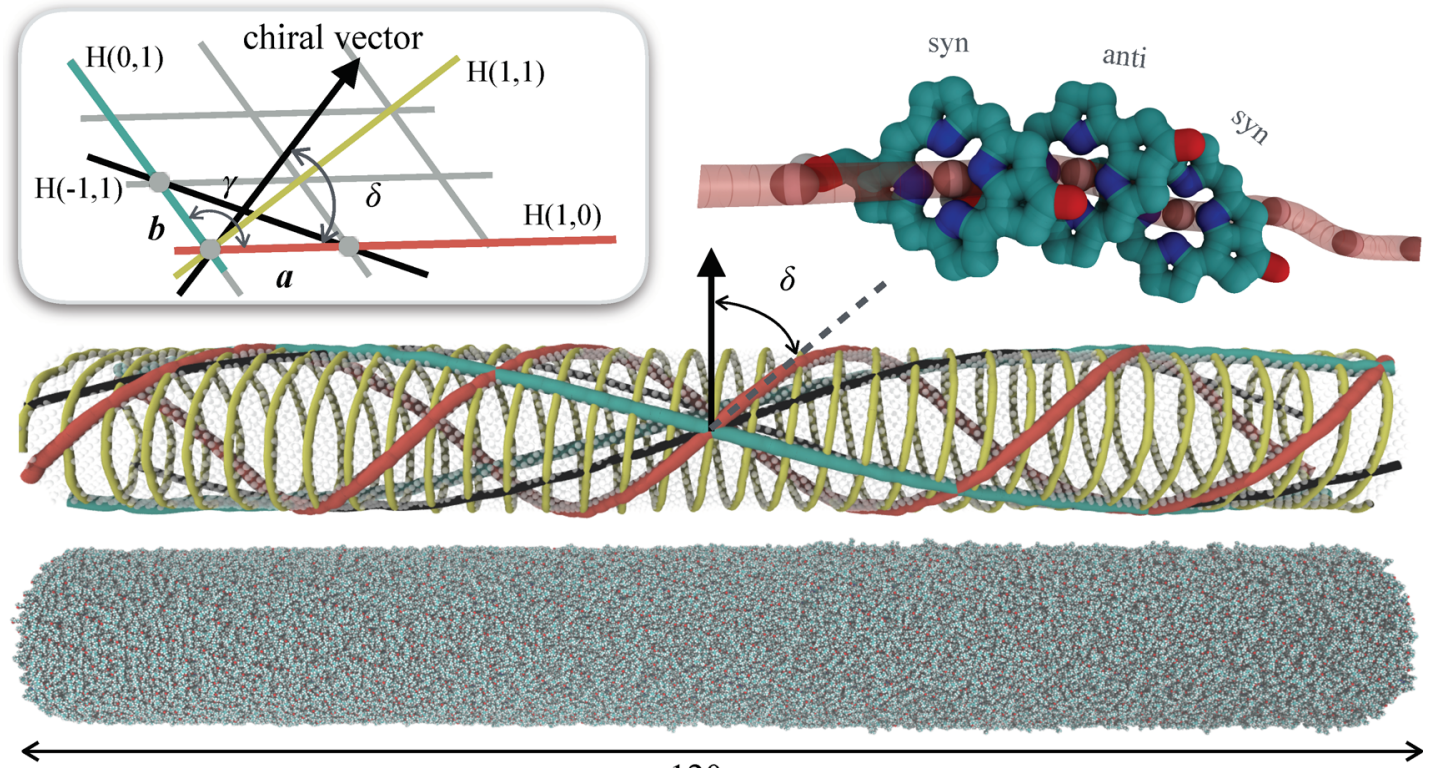

$120 \mathrm{~nm}$

Figure 1. Different representations of the preassembled tube structure for $\delta=49.6^{\circ}$ after $1 \mathrm{~ns} \mathrm{MD}$ simulation at $300 \mathrm{~K}$. Top, left: Schematic illustration, showing the 2D Bravais lattice and the $H(1,0)$ (red), $H(0,1)$ (green), $H(1,1)$ (yellow) and $H(-1,1)$ (black) helical families as well as the chiral vector. Top, right: Schematic illustration showing the arrangement of alternating syn and anti molecules along the $H(1,0)$ helical family, which connects the Mg-moieties. Middle: The main helical families in the 3D tube, obtained from connecting Mg-moieties. Two helices for each family are shown, with the same coloring as in the schematic image at top, left. Bottom: Molecular view of the tube. For WT chlorosomes, the lattice parameters are determined as $\left(1.48,0.98,124.3^{\circ}\right)$ from $\mathrm{MD}$ simulation of $\mathrm{BChl} c$ pigment aggregates; for $b c h \mathrm{QRU}$ chlorosomes, the lattice parameters are determined as $\left(1.46,0.93,122.5^{\circ}\right)$ from $\mathrm{MD}$ simulation of $\mathrm{BChl} d$ pigment aggregates.

plant photosynthesis, where the chlorophylls in photosystem I and II have the same color despite widely different redox potentials. $^{35}$ On a more fundamental level, delocalized eigenstates like the ones that have been observed in chlorosomes can only exist when transitions are near resonant, meaning that the difference in monomer excitation energies between two molecules must be much smaller than the difference in their electronic coupling. ${ }^{9}$ We thus set the monomer excitation energies for all molecules to the same constant value, i.e., $\nu_{i}(\mathbf{R})=\nu$, meaning that we assume that any fluctuation with respect to this reference energy $\nu$ on the diagonal are negligible compared to the fluctuations of the energy in the off-diagonal terms. ${ }^{36}$

It is nevertheless common to represent the unknown variations of the internal and external conditions for each pigment molecule in a chlorosome by adding an effective fluctuating contribution to $\nu_{i}^{0}$, usually drawn from a distribution with Gaussian statistics and adjustable width $\sigma .{ }^{6}$ Although $\sigma$ could be chosen to match calculated fluctuations of the monomer excitation energy for significantly reduced systems, ${ }^{37}$ it is usually tweaked to match experimental fwhm's, which are known to reflect both static and dynamic disorder. Furthermore, adding a weak to intermediate correction to the site energy will only results in weak mixing of excitonic wave functions and a mild spreading of the peak spectra. ${ }^{6}$ Besides our original arguments, see previous paragraph, we note that adding a Gaussian broadening correction to the monomer excitation energy, ${ }^{6,37}$ has the clear disadvantage of blurring or even destroying the effect of cooperativity as included into $J_{i k}(\mathbf{R})$ via the MD information.

\section{RESULTS AND DISCUSSION}

The first aim of this study is to develop a systematic and rigorous procedure for translating the characterization data, which have been extracted at various levels of resolutions, into a molecular model for chlorosomes. A key challenge in this process is that we can not represent all experimental factors in our computational model. In particular, the structure of natural chlorosomes encompasses heterogeneity in size, in overall shape, and in composition; a cryo-EM image of a chlorosome may experience small tilts out of plane of imaging. ${ }^{38}$ All such factors modulate the signal intensity along layer lines in the diffraction pattern of a cryo-EM image, as can be seen from the single, weak layer lines in the noisy spectra for both WT and $b c h Q R U$ chlorosomes. ${ }^{2}$ For this reason, we must thus take care in identifying the characteristics that Fourier responses calculated for our preassembled tubes should capture beyond the layer line position $1 / d$, which is an indicator of a correlation length, in the experimental power spectra. In particular, the geometrical analysis presented here enables further fine-tuning if needed, for instance when additional experimental information becomes available in the future. Since the characteristics of dynamic disorder were found to be conserved under varying tube chirality, the second aim, i.e., evaluating the 
(a) optical spectra observed in experiments

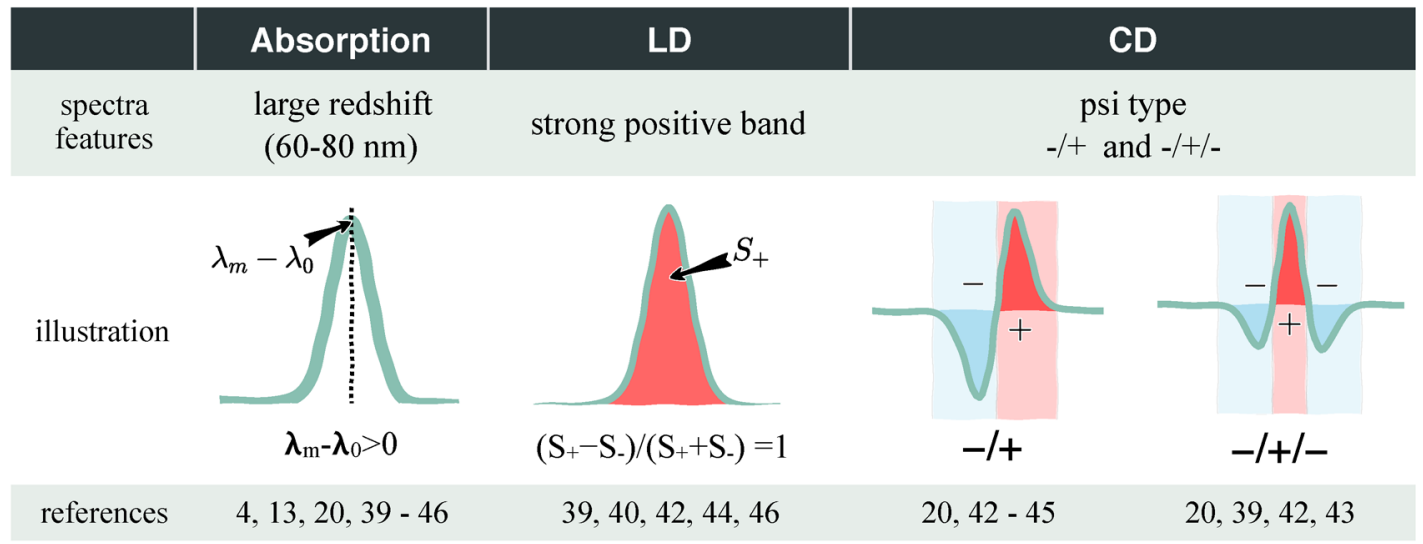

(b) additional types of optical spectra

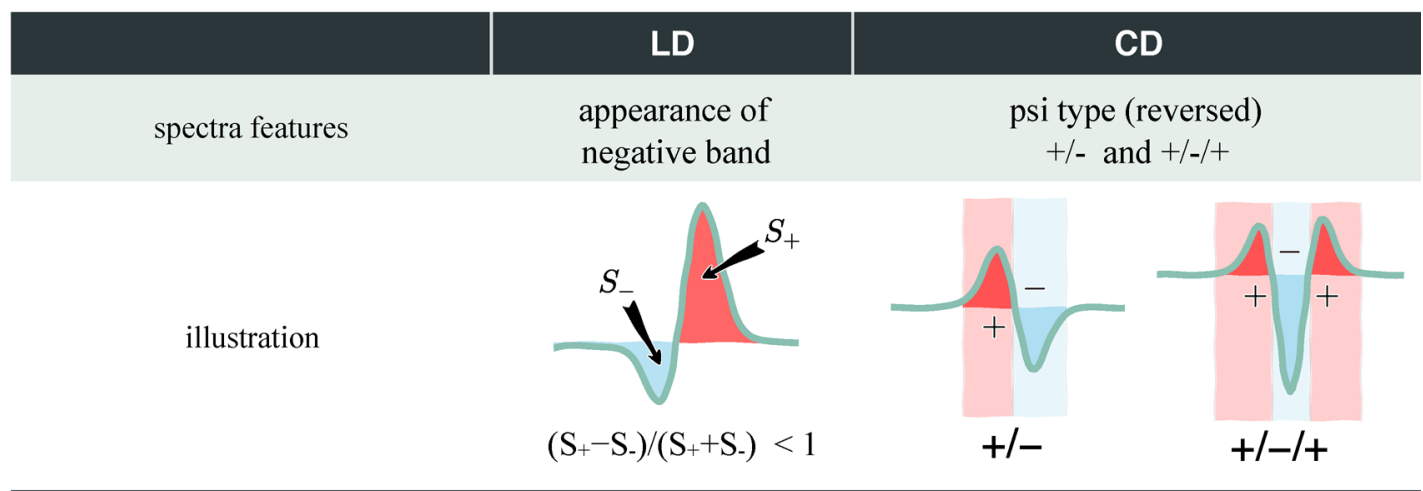

Figure 2. (a) Schematic summary of key features of the experimental absorption, linear and circular dichroism spectra measured for chlorosomes. Main features, key properties and the corresponding literature reference are provided. (b) Sketches of additional optical spectra resulting from our calculations, for which no or only few experimental counterparts have been identified.

interplay of quantum and classical degrees of freedom, can be considered via a particular case.

An important aspect of this procedure is to generate tubes that are representative for the dynamic disorder that plays a role in actual chlorosomes. We previously found that hydrogen bonding heterogeneity and head-head rotations are characteristic for the tubular chiral assemblies that are spontaneously formed after a relatively short $1 \mathrm{~ns} \mathrm{MD}$ simulation at $T=300$ K. ${ }^{5}$ Following our geometrical analysis, tube structures corresponding to matching $\delta$ are thus used as input for MD simulations in order to introduce the disorder that is needed for a realistic comparison to experimental diffraction and optical spectra.

3.1. Extended Geometrical Analysis. We have extended the geometrical analysis developed in earlier work ${ }^{5}$ to consider a complete set of candidate helical families and the whole range of the chiral angle $\delta \in\left[0^{\circ}, 180^{\circ}\right]$; see Figures S1 and S2 and details in the Supporting Information. As before, we may employ this geometrical framework to link layer line positions $1 / d$ in the diffraction pattern, i.e., $1 / d=1 / 1.25 \mathrm{~nm}^{-1}$ for WT and $1 / d=1 / 0.83 \mathrm{~nm}^{-1}$ for $b c h Q R U$ chlorosomes, to a chirality of the tube. To ensure internal consistency, we selected dimensions of the dimeric unit cell $(a, b, \gamma)$ that were previously extracted from MD results for assemblies composed of pure BChl $c$ and $d .^{5}$ It should be noted that, since these unit cell parameters were determined as averages and feature standard deviations in the $0.03-0.05 \mathrm{~nm}(a$ or $b)$ and $2-4^{\circ}(\gamma)$ range, see Supporting Information in $\mathrm{Li}$ et al., ${ }^{5}$ each reported $\delta$ is in fact part of a tiny window of appropriate angles associated with that $\delta$. Table 1 summarizes the chiral angles $\delta$ that exactly match $1 / d$ for the considered unit cells of WT or bchQRU chlorosomes. In the standard classification, which relates to the orientation of syn-anti stacks to the tube long axis, ${ }^{2}$ i.e., perpendicular for $\delta=0^{\circ}$ and parallel for $\delta=90^{\circ}$, assemblies for $\delta \in\left\{2.2^{\circ}, 19.8^{\circ}, 160.2^{\circ}\right\}$ can be seen as primarily perpendicular, while they are primarily parallel for $\delta \in$ $\left\{80.0^{\circ}, 94.3^{\circ}, 108.9^{\circ}, 112.3^{\circ}\right\}$. Intermediate values, i.e., for $\delta \in$ $\left\{27.6^{\circ}, 42.3^{\circ}, 49.6^{\circ}, 130.4^{\circ}, 136.3^{\circ}\right\}$, relate to neither parallel nor perpendicular stacks.

Next, we constructed tube structures for the matching $\delta$ in Table 1, see Method section for details, and found that they are all stable upon $1 \mathrm{~ns} \mathrm{MD}$ simulation at $T=300 \mathrm{~K}$. The preassembled structure for one matching chiral angle, i.e., $\delta=$ $49.6^{\circ}$ is shown in Figure 1. Visual inspection shows that it is hard to identify atomic detail, making this representation illustrative for the robustness of such preassembled structures to thermal dispersion, in particular when concentrating on the large scale characteristics such as the overall chirality. Later, in section 3.3, we will analyze structural properties more quantitatively via the positions of the $\mathrm{Mg}$ lattice sites and orientations of the $\mathrm{BChl}$ head groups. Consequently, we performed Fourier transforms on these preassembled structures, both before and after $\mathrm{MD}$, to determine the power spectra for their EM mimics; see Figure S3 and Figure S4 in the 


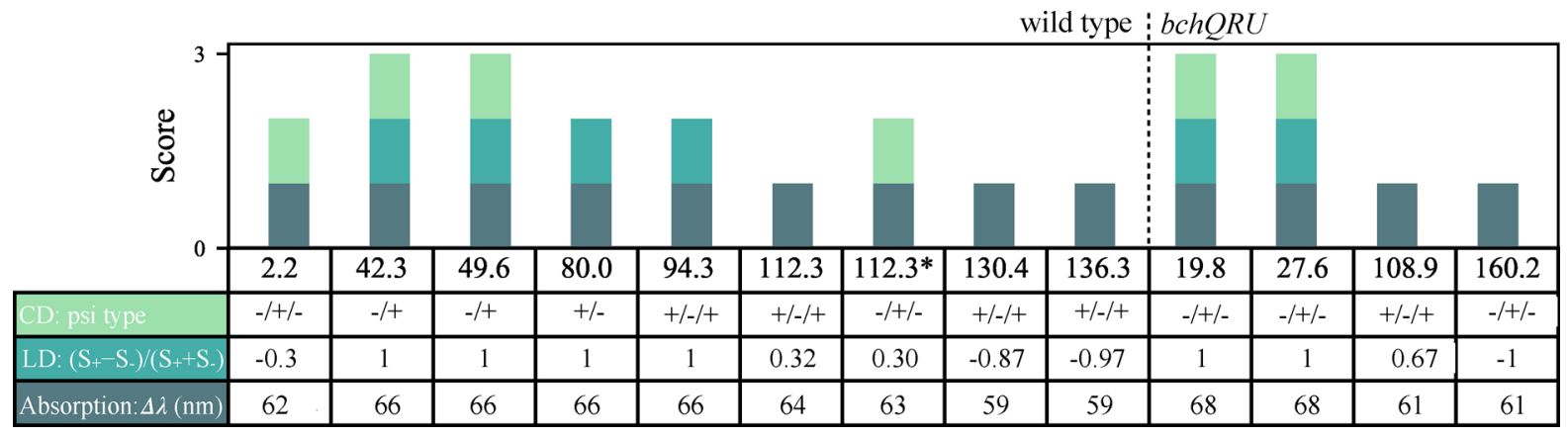

Figure 3. Overview of the spectral characteristics for all tube structures with matching chiral angles $\delta$. Stick spectra were calculated for all tubes after 1 ns MD simulation at $300 \mathrm{~K}$, and characteristics were determined for the spectra that resulted from convolution of the stick spectra with a Gaussian with fwhm equal to $200 \mathrm{~cm}^{-1}$. Colored scoring bars on top of the table show which characteristics of the simulated spectra agree with the experimentally observed spectral properties.

Supporting Information. Examining these diffraction spectra allows us to validate our geometrical analysis and to qualitatively compare the characteristics of the target layer line signals to experimental data. ${ }^{2} \mathrm{We}$ find that important characteristics of the experimental layer line, i.e. signal intensity close to the meridian, ${ }^{2}$ are reproduced by the target layer line for $\delta \in\left\{112.3^{\circ}, 130.4^{\circ}, 136.3^{\circ}\right\}$ for WT and $\delta \in\left\{19.8^{\circ}, 160.2^{\circ}\right\}$ for bchQRU type chlorosomes; see Figure $S 3$ for all the diffraction patterns for tube structures after MD simulations.

In the final step of the procedure, optical spectra for all tube structures, after MD simulation $(T=300 \mathrm{~K})$, are compared with the optical spectra measured in experiments. We note that the tube size is important for spectral calculations, as optical spectra for tubular assemblies have been reported to be size sensitive, ${ }^{6,10,20}$ especially the shape of the CD spectra, which may change when the tube length crosses a so-called critical value. Tang et al. ${ }^{20}$ particularly focused on the relation of the critical value to the tube diameter and tube length, for an assembly with chiral angle $\delta=90^{\circ}$, and determined a critical length of about $90 \mathrm{~nm}$ for a tube diameter of $15 \mathrm{~nm}$. Here, we selected a diameter of $15 \mathrm{~nm}$ and considered optical signals for tubes up to $120 \mathrm{~nm}$ length and all matching $\delta$ to evaluate this length dependence. Based on the finding that spectral features show no notable variation in the range from 60 to $120 \mathrm{~nm}$, see Figures S5 and S6, we further concentrate our analysis on tubes of $120 \mathrm{~nm}$, which is comparable in length to a natural chlorosome. A single tube of this dimension is composed of over one million atoms.

3.2. Calculated Optical Spectra. Optical spectroscopy data have been collected from both WT and mutant chlorosomes, including absorption, ${ }^{4,13,20,39-46}$ linear dichroism $^{39,40,42,44,46}$ and circular dichroism..$^{20,39,42-45}$ Absorption is most frequently measured and found rather insensitive to the chirality of the assembly, whereas the LD and especially CD spectra are more distinctive of particular chirality and therefore useful for further selection of specific $\delta .{ }^{47}$ The observed spectral features are summarized in Figure 2, which shows that two types of CD spectra have been found for Chloroflexus aurantiacus and Chlorobium tepidum WT or mutant chlorosomes: type II $(-,+)$ in the notation of Gribenow et al., ${ }^{42}$ and a mixed-type $(-/+/-)$ that can be interpreted as a linear combination of type I $(+/-)$ and type II. Although type I spectra have also been reported for chlorosomes on two occasions, ${ }^{42,48}$ we restrict our matching procedure to the two dominant spectra. Since reversed curving may switch the sign of CD spectra, it has been suggested that mixed-type CD spectra can be assigned to a mixture of tubular complexes with similar BChl packings but opposite curving directions. ${ }^{2}$ In this section, however, we concentrate on calculating optical properties for single tubes, thereby adopting earlier notions that multiple cylinders can be treated as uncoupled systems for the purpose of modeling optical spectra. ${ }^{49,50}$ Our results will particularly show that a single tube is sufficient to generate a mixed-type CD spectrum. An overview of all simulated spectra is provided in the Supporting Information, see Figure S7, and a comparison of calculated and experimental properties is provided in Figure 3.

Absorption spectra are characterized by a shift $\lambda_{m}-\lambda_{0}$, with $\lambda_{m}$ the absorption peak position of the aggregate and $\lambda_{0}$ of the monomer, which can be either positive (red shift, J-aggregate) or negative (blue shift, H-aggregate). ${ }^{51}$ As mentioned previously, $\lambda_{0}$ is set to $15390 \mathrm{~cm}^{-1}$ or $650 \mathrm{~nm}$. $^{6}$ Chlorosomes are J-aggregates associated with a large red-shift, i.e., $\lambda_{m}-\lambda_{0}=$ $60-80 \mathrm{~nm},{ }^{3}$ and indeed an equivalent red shift is observed in all calculated absorption spectra for our tube structures, see Figure 3. The insensitivity of the shape of the absorption band to tube chirality shows that the $Q_{y}$ absorption band of chlorosomes is mainly determined by the short-range order of syn-anti dimers and only weakly reflects longer-ranged structural properties like supramolecular chirality. We note that a significantly smaller red shift is produced when switching to the TrEsp treatment for the electronic coupling, although this method was tested more accurate than the PDA. ${ }^{18}$ In particular, calculations with TrEsp for our tubular assemblies $\left(\delta=49.6^{\circ}\right.$ and $\left.112.3^{\circ}\right)$ resulted in a red shift $<10 \mathrm{~nm}$, a finding that is consistent with previous work. $^{21}$

Linear dichroism reveals the order of alignment more directly, as strong LD signals are expected only for ordered systems. ${ }^{47}$ Indeed, strong signals are observed from the calculated LD spectra for all tube structures, see Figure S7. The sign of the LD signal is determined by the absorbance of light polarized parallel and perpendicular to the tube axis, $\mathrm{LD}=$ $A_{\|}-A_{\perp}$, meaning that a negative/positive signal appears when absorption along the tube axis is weaker/stronger than that orthogonal to the tube axis. We introduce the relative difference $f_{l d}=\left(S_{+}-S_{-}\right) /\left(S_{+}+S_{-}\right)$to better characterize these LD spectra, with $S_{+}$and $S_{-}$being the integrated area of the positive and negative parts of the LD signals, respectively. Three types of LD-spectra are observed; see Figure 3: (i) strictly positive, with $f_{l d}=1$, as in the experimental LD spectra, (ii) signal displaying a small negative peak in the short-wavelength range and $f_{l d}<1$, and (iii) strictly negative signals, with $f_{l d}=-1$. Matching these features to the experimental LD spectra limits 
the chiral angles to $\delta=42.3^{\circ}, 49.6^{\circ}, 80.0^{\circ}$, and $94.3^{\circ}$ for WT chlorosomes and $\delta=19.8^{\circ}$ and $27.6^{\circ}$ for $b c h Q R U$, respectively. We exclude $\delta=112.3^{\circ}$ and $108.9^{\circ}$ since $f_{l d}<1$, even though $S_{+}$ $\gg S_{-}$.

The circular dichroism (CD) spectrum is most sensitive to the aggregate structure; in particular, a special, psi-type $C D$ spectrum is expected for chiral aggregates. ${ }^{47}$ In total four types of psi-type CD spectra are calculated for our preassembled tubes: type I $\left(+/-, \delta=80.0^{\circ}\right)$, type II $(-/+, \delta \in$ $\left.\left\{42.3^{\circ}, 49.6^{\circ}\right\}\right)$, and two mixed-types, namely $(-/+/-)$ for $\delta$ $\in\left\{2.2^{\circ}, 19.8^{\circ}, 27.6^{\circ}, 160.2^{\circ}\right\}$ and $(+/-/+)$ for $\delta \in$ $\left\{94.3^{\circ}, 108.9^{\circ}, 112.3^{\circ}, 130.4^{\circ}, 136.3^{\circ}\right\}$. The results confirm that mixed-type $C D$ spectra are found even for single tubes with well-defined chiralities, and it shows that this type can originate from mixing various contributions of the helical families that are present in a tube, see Figure 1 . Since only types $(-/+)$ and $(-/$ $+/-)$ are consistently measured for chlorosomes, ${ }^{20,42}$ this further reduces the number of matching $\delta$. Moreover, this is the first time that type II $(-/+)$ is calculated for tubular assemblies that approach realistic chlorosomes dimensions. Extending the tube length for $\delta=49.6^{\circ}$ even further, to $300 \mathrm{~nm}$, shows that the type II CD signal is persistent. The novelty of this finding can be explained by the fact that previous computational studies of spectral properties only considered $\delta \in\left\{0^{\circ}, 90^{\circ}\right\}$.

In combination, our procedure suggests that only a tiny range of the chiral angles in Table 1 provide relevant optical spectra: for WT chlorosomes, $\delta=42.3^{\circ}$ or $49.6^{\circ}$, and $\delta=19.8^{\circ}$ or $27.6^{\circ}$ for $b c h Q R U$ chlorosomes; see the score bars in Figure 3 for details. We find that the meridian nature of the layer line signal calculated for the tube for $\delta=19.8^{\circ}$ agrees well with the experimental layer line characteristics, which provides converging evidence that $\delta=19.8^{\circ}$ is a unique solution for $b c h Q R U$ chlorosomes that are rich of $\mathrm{BChl} d$. We note that this angle is consistent with the previously extracted $\delta=19.1^{\circ}$ for pure BChl $d$ aggregates that are formed by spontaneous curvature after $1 \mathrm{~ns}$ of $\mathrm{MD}$, and with the perpendicular-type structural model proposed previously. ${ }^{2}$ For WT chlorosomes, although the matching $\delta$ values appear to be consistent with the angles extracted from $\mathrm{MD},{ }^{5}$ the experimentally observed layer line characteristics, i.e., a meridian signal in the diffraction spectrum, is not reproduced. Moreover, for the candidate $\delta=112.3^{\circ}$ that does qualitatively agree with these characteristics, both the LD and CD signals are inconsistent. Since our curvature direction and average unit cell dimensions were both adopted from MD simulations, ${ }^{5}$ it makes sense to consider how these factors affect the optical spectra.

If one envisions tubes to be generated by rolling up planar structures, it is easy to understand how reversing the direction of rolling will lead to a reversal of chirality. Current computational studies of spectral behavior ${ }^{6,7,10,11,20,21}$ consider simple dipolar lattices that lack any molecular detail, which clarifies why this subtle issue has never been addressed. So far, our curving direction is selected such that syn-tails always direct toward the concave, inner side of the tube. ${ }^{5}$ To investigate the role of the overall curving direction in optical spectra, we have assembled a tubular structure for $\delta=112.3^{\circ}$ and a reversed curving direction, i.e., with anti-tails toward the inside of the tube. This situation is labeled with an asterisk, $\delta=112.3^{*}$, to distinguish it from the standard case.

From a $1 \mathrm{~ns} \mathrm{MD}$ simulation at $T=300 \mathrm{~K}$, we find that reversing the curving direction does not notably affect the stability of the molecular and supramolecular packing. As expected, the $C D$ spectrum can be seen to reverse, from
$(+/-/+)$ for $\delta=112.3^{\circ}$ to $(-/+/-)$ for $\delta=112.3^{*}$, see Figure 3 , i.e., consistent with the experimental CD spectra, confirming the sensitivity of the CD-spectra to chirality. However, since the OD and LD spectra do not change upon reversing the curving direction, the negative peak that is present in the shortwavelength side of the calculated spectrum for $\delta=112.3^{\circ}$, and thus also for $\delta=112.3^{*}$, does not agree with the experimental LD spectra. It should be noted, however, that the characteristics of the LD spectrum vary in a range around $\delta=112.3^{\circ}$, with $f_{l d}$ changing from $f_{l d}=1$ for $94.3^{\circ}$ to $f_{l d}=-1$ for $136.3^{\circ}$, see Figure S7. Further testing identified matching $\mathrm{OD}, \mathrm{LD}$, and $\mathrm{CD}$ spectra for $\delta=105^{*}$, which is only slightly smaller than $\delta=$ $112.3^{*}$ and just within the window of chiral angles around $\delta=$ $112.3^{*}$ that are consistent with the earlier identified $0.05 \mathrm{~nm}$ margin of the unit cell parameter $a$. This is converging evidence that $\delta=105^{*}$ is the proper chiral angle for WT chlorosomes.

As a final remark, it has always been difficult to explain the uncontrolled variation of measured CD-spectra for WT chlorosomes in terms of the underlying structure, with both type II and mixed-types encountered for the same WT chlorosomes in different measurements. The benefit of considering all $\delta$ in Table 1 is that it allows us to investigate the sensitivity of the LD and CD spectra to the tube chirality. For instance, a transition from a mixed-type to a type II CD spectrum transition is found somewhere between $\delta=27.6^{\circ}$ and $42.3^{\circ}$. Such a small variation of the base tube chirality may well be a consequence of $\mathrm{BChl}$ compositional heterogeneity or particular growth conditions. Moreover, the highly efficient function of both WT and $b c h Q R U$ chlorosomes is a clear sign that performance is conserved for tubular assemblies that feature different chirality but share important properties on a local structural level, something that is confirmed by a detailed comparison of static and dynamic disorder for a number of different $\delta$ in the following section 3.3. For this reason, in sections 3.4-3.6 where we investigate the interplay between dynamic disorder and electronic properties, we illustrate a single chiral angle, i.e., $\delta=49.6^{\circ}$, for which the optical spectra match the experimental data.

3.3. The Origin of Disorder. Next, we focus on the disorder in the tube structures after MD simulation. We do this by considering various structural properties of the preassembled tubular assemblies as well as how these properties are affected by the thermal motion. Table S1 summarizes the structureaverage lattice parameters $(a, b, \gamma)$ and two large-scale structural properties, i.e., the chiral angle $\delta$ and the tube radius $R$ derived from the best tube fit. When building assemblies following the procedure described in Methods, lattice parameters are set to $(a, b, \gamma)=\left(1.48,0.98,124 \cdot 3^{\circ}\right)$. All structural properties are calculated from the $\mathrm{Mg}$ positions in the assembly at various steps of the simulation procedure: after preparation and energy minimization at $T=0 \mathrm{~K}$, after $1 \mathrm{~ns} \mathrm{MD}$ at $T=50 \mathrm{~K}$ and $T=300$ $\mathrm{K}$.

We find that lattice parameters are generally conserved within tiny margins, and the same conclusion can be drawn for the input chiral angle $\delta$, even for $T=300 \mathrm{~K}$. Although the rootmean-square deviations from the fitted tube, RMSD ${ }_{\text {fit }}$ increase with temperature, the assembly remains cylindrical with RMSD ${ }_{\text {fit }} \leq 0.3 \mathrm{~nm}$ for all $\delta$ of BChl $c$. Apparently the structure does not need to resolve possible frustrations resulting from manual assembly by adapting its unit cell or assuming a noncylindrical shape. Visual inspection, however, shows that tube assemblies of $\mathrm{BChl} c$ are slightly more regular than for $\mathrm{BChl} d$, which is in agreement with the findings for spontaneously self-assembled 


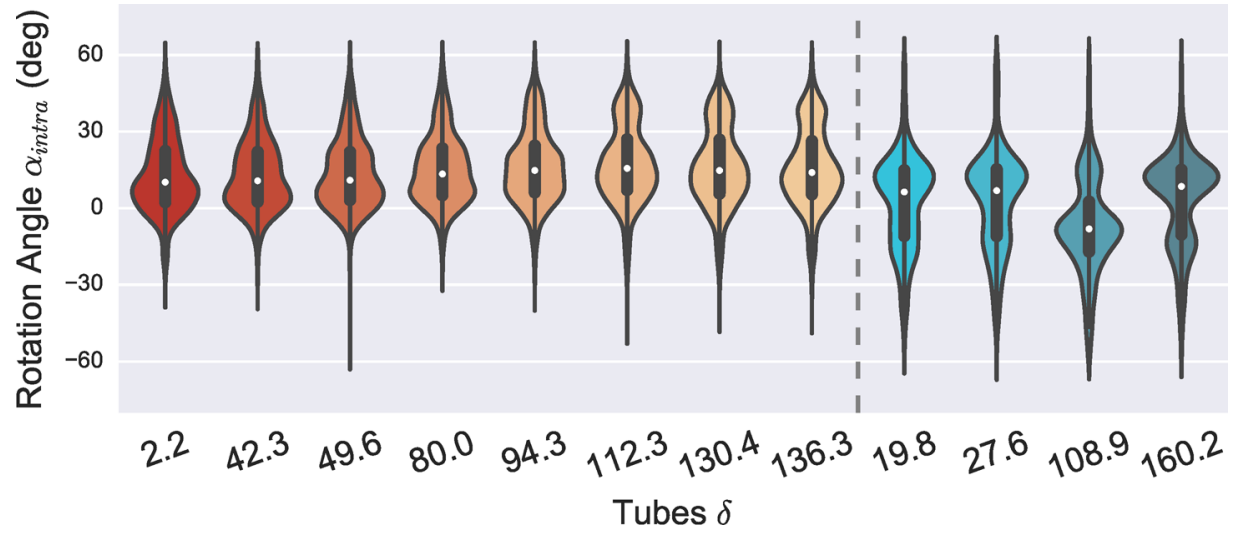

Figure 4. Sampling distribution for the relative head-head rotation angle between syn and anti molecules BChl, $\alpha_{\text {intra }}$ for the tube obtained after $1 \mathrm{~ns}$ $\mathrm{MD}$ simulation at $300 \mathrm{~K}$. The distribution is calculated for different chiral angles $\delta$ on the horizontal axis, and the dashed line separates the matching chiral angles for BChl $c$ and $d$. We refer to the Supporting Information, Figure S8, for the distribution of $\alpha_{\text {inter }}$.

structures in $\mathrm{Li}$ et $\mathrm{al}^{5}$ and explains the small increase in $\mathrm{RMSD}_{\text {fit }}$ for the latter. On basis of this analysis, which mainly considers the central $(\mathrm{Mg})$ position of the BChl molecules, we conclude that the static disorder in all considered assemblies is fairly limited.

As in our previous study for spontaneous assembly, ${ }^{5}$ we identify dynamic disorder in the form of a rotational degree of freedom for BChl head groups, so the tubular assemblies can indeed be seen as a rotor phase. ${ }^{52}$ Whereas the static crystal-like order is quite well conserved, see Table S1, the distribution of relative intra- and intermolecular head-head rotation angles, $\alpha_{\text {intra }}$ and $\alpha_{\text {inter }}$ is broad, see Figure 4 and Figure S8, and their sampling distributions are comparable to the ones previously observed for spontaneously curving structure. Likewise, the hydrogen bonding heterogeneity that was found to accompany this rotational motion ${ }^{5}$ is present in all tube considered structures after MD simulation. The previously determined hydrogen-bonding saturation ratio, i.e. the fraction of hydrogen bonds present in the structure that are actually formed, ${ }^{5}$ is appropriately reproduced for preassembled tubes simulated at $T=300 \mathrm{~K}$, namely 0.74 for $\mathrm{BChl} c\left(\delta=49.6^{\circ}\right)$ and 0.87 for $\mathrm{BChl} d\left(\delta=19.8^{\circ}\right)$. Since the rotational dynamics is clearly not affected by assembling BChl in a tube structure, it is convincing evidence that they are stable structures. Moreover, it shows that dynamic disorder is much more pronounced in such assemblies than static disorder, in agreement with our choices in the Frenkel Exciton Hamiltonian. The limitation of static disorder is also justified by NMR and optical spectra experiments. ${ }^{2,7}$

3.4. Localization of Excitonic States by Disorder. In our treatment, disorder that is present in the tubular assemblies, in the form of molecular deformations, vibrating molecular lattices, and rotating head parts of BChl, is reflected in the off-diagonal terms $J_{i k}$ of the Frenkel Exciton Hamiltonian matrix. Next, we concentrate on how such disorder affects the spatial distribution of relevant exciton states. Since MD simulations account for correlations within the assembly, it represents a substantial advance compared to previous studies that lack such cooperativity. ${ }^{6}$ We will compare the spatial aspects of exciton states for three degrees of disorder, i.e., order at $0 \mathrm{~K}$, mild disorder at $50 \mathrm{~K}$ and moderate disorder at $300 \mathrm{~K}$, and for $\delta=49.6^{\circ}$ that was selected for as an illustrative case.

First, we analyze the distribution of electronic coupling strengths $J_{i k}$, see Figure 5. Since $J$ is a quickly decaying function, we have used a cutoff of $1.5 \mathrm{~nm}$. Five distinct peaks can be

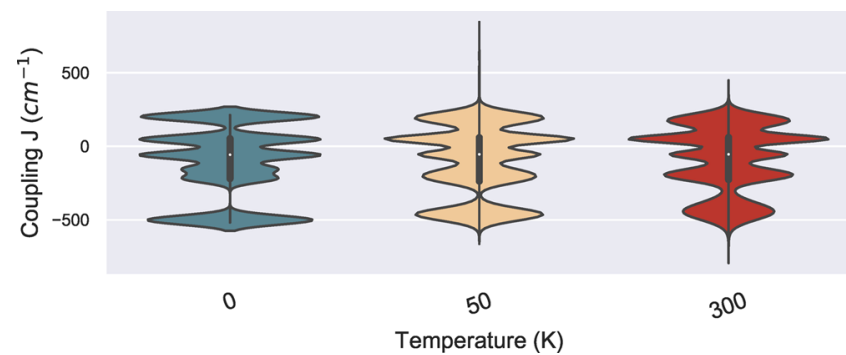

Figure 5. Distribution of coupling strengths $J_{i k}$ for $\delta=49.6^{\circ}$ and a 120 $\mathrm{nm}$ tubular structure at $0 \mathrm{~K}$, i.e., prepacked after energy minimization, 50 and $300 \mathrm{~K}$ after $1 \mathrm{~ns} \mathrm{MD}$ simulation. The strengths are calculated using a cutoff distance of $1.5 \mathrm{~nm}$.

observed for all considered $T$, reflecting the earlier finding that chirality is conserved under thermalization. Distinct bands stem from molecular packing along main helical families in the tube, e.g., the strong coupling peak around $-500 \mathrm{~cm}^{-1}$ corresponds to syn-anti packing along $H(1,0)$, and has a major contribution to the large red-shift of the $Q_{y}$ band. Due to thermal fluctuations, broadening and smoothing of the coupling strength distributions is observed in the disordered states (50 and $300 \mathrm{~K}$ ).

The conservation of the bands in the coupling distribution under thermalization is in line with comparable spectral features for ordered and disordered situations, see spectral plots in Figure S9, and consistent with the observation of Prokhorenko et al. $^{6}$ that the effect of adding off-diagonal noise is equivalent to Gaussian broadening of calculated stick spectra. The insensitivity of the main spectral features to molecular motion justifies the focus on perfect lattice models in previous studies of spectral signals. ${ }^{6,7,10-12}$ However, when one is also interested in details like the spatial distribution of exciton states that correspond to stick-signals in optical spectra, it is advantageous to consider structure with realistic disorder from a physical point of view. ${ }^{8,53}$

Furthermore, profound knowledge of the spatial distribution of an exciton is essential for a fundamental understanding of the energy transfer in chlorosomes. An important feature of Frenkel excitons is that, due to the strong electronic coupling between monomers, exciton states are usually distributed over more than one monomer, which is described as the delocalization of an exciton. ${ }^{8,54}$ Such delocalization is a static property, and should not be confused with exciton transport. In recent years, $2 \mathrm{D}$ 
(a)
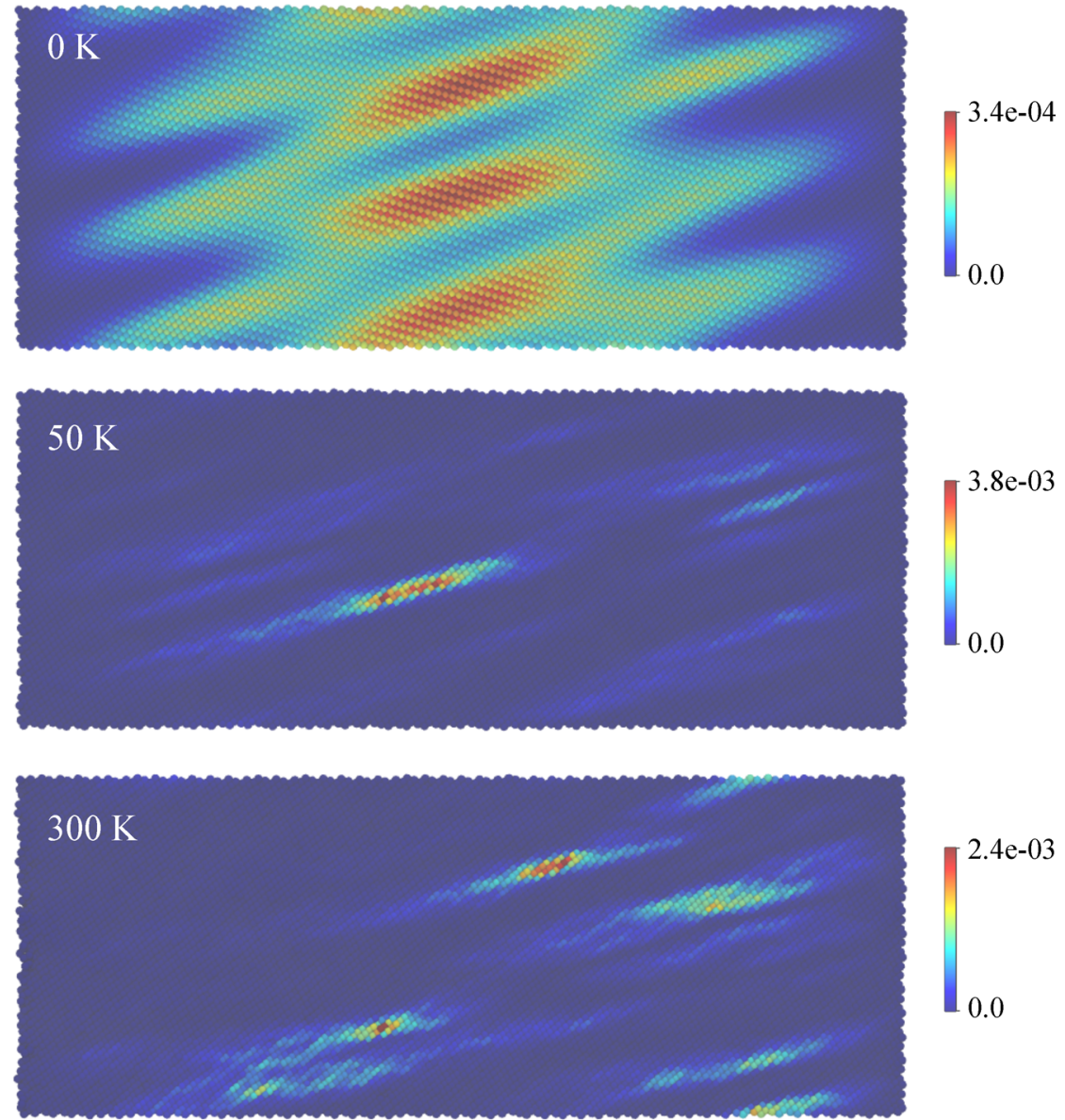

(b)

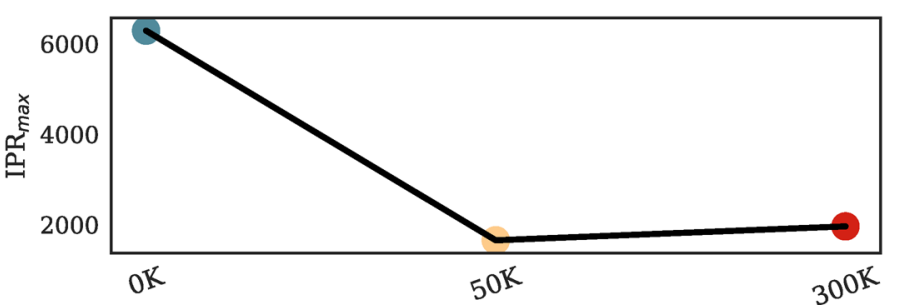

Figure 6. Spatial distribution of the exciton states with the highest oscillation strength $\psi_{\max }$ in a $120 \mathrm{~nm}$ long tube for $\delta=49.6^{\circ}$. (a) From top to bottom: $\psi_{\max }$ for different disorder within the tube: ordered state, labeled as $0 \mathrm{~K}$, and disordered states after $1 \mathrm{~ns}$ MD simulation, labeled as 50 and $300 \mathrm{~K}$. The localization of $\psi_{\max }$ is quantified by the IPR; see part $\mathrm{b}$. Tube structures have been unrolled to planes for the purpose of visualization; see Figure S10 for corresponding 3D tubes.

spectroscopy has identified several cases of intricate dynamic modulation of exciton transport by so-called coherent beatings, and a basic description for the corresponding line shape dynamics for chlorosomes has recently been proposed. ${ }^{13,55,56}$ This model assumes that tubes feature a patchwork of coherent domains in which excitons adopt wave-like behavior, while excitons efficiently hop between coherent domains of very similar energy in an incoherent manner. ${ }^{13,55}$ Yet, existing theoretical and computational studies have not been able to further rationalize this mechanism. In particular, disregarding structural disorder on a molecular level, by considering (semi)crystalline structures, ${ }^{6,7,10-12,19-21,37}$ overestimates the delocalization of excitonic states ${ }^{6,10,11}$ and is therefore fundamentally unsuited for studying such effects.

Our current treatment enables us to conclude that disorder indeed suppresses the delocalization of excitons. In Figure 6, the spatial distribution of the exciton state $\psi_{\max }$, corresponding to the largest oscillation strength in the absorption spectrum, is visualized for different disorder $(0,50$, and $300 \mathrm{~K})$ of the tube structure. In the ordered case, see Figure $6 a, \psi_{\text {max }}$ can be seen highly delocalized, a finding that is consistent with previous studies for regular structures. ${ }^{6,10,11}$ Introducing disorder due to thermal motion has a localizing effect on the exciton domain size, with the domain of significant exciton density shrinking to $O(10)$ monomers for $T=300 \mathrm{~K}$, see Figure 6a, as estimated in an experimental investigation. ${ }^{23}$ Most importantly, the spatial distribution of the exciton state can be seen to change from extended striped domains, see Figure $6 \mathrm{a}$, top, for $T=0 \mathrm{~K}$, to clusters of tiny domains of high exciton density, see Figure 6a, bottom, for $T=300 \mathrm{~K}$.

The (de)localization of states can be quantified by their inverse participation ratio (IPR), see the Methods section for details. Denoting the IPR for the state with the largest oscillator strength as $\mathrm{IPR}_{\text {max }}{ }^{26}$ we find an abrupt decrease from a high 


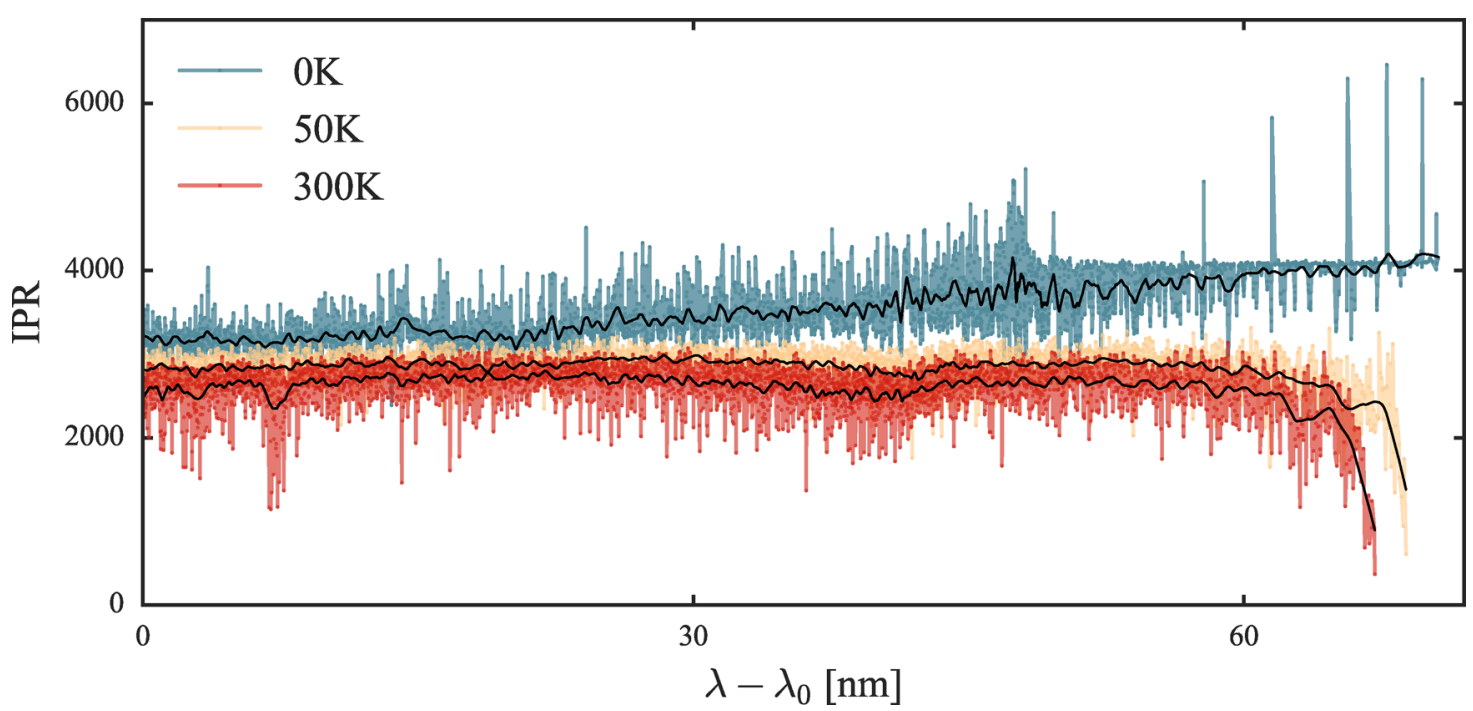

Figure 7. Inverse participation ratio (IPR) for all exciton states with energy lower than the monomer excitation energy $15390 \mathrm{~cm}^{-1}$. In this range, states belong to the red-shift absorption band and satisfy $\lambda-\lambda_{0}>0$. The setup is the same as in Figure 6, i.e., a $120 \mathrm{~nm}$ long tube for $\delta=49.6^{\circ}$ and different temperatures.

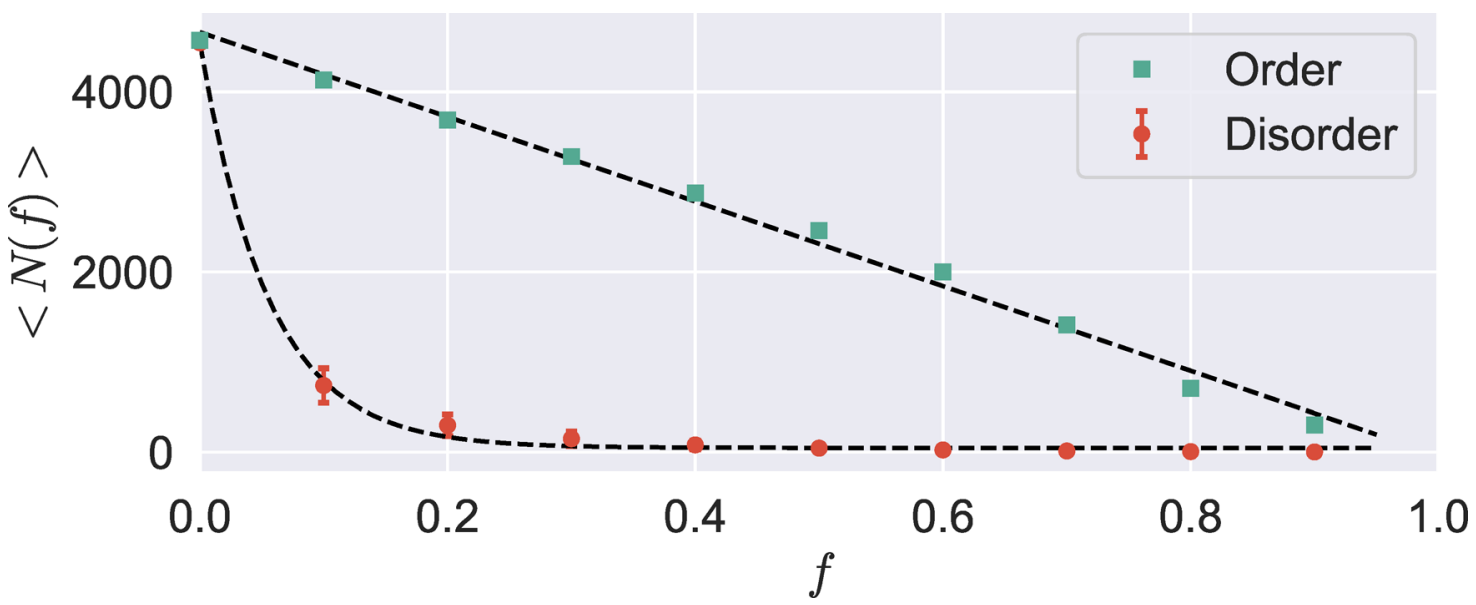

Figure 8. Average number of molecules $\langle N(f)\rangle$ versus fraction $f$ for the state with the largest oscillation strength in a $60 \mathrm{~nm}$ long tube for $\delta=49.6^{\circ}$. Green symbols represent the data for an ordered structure after energy minimization, and red shows averages over 1 ps $\mathrm{MD}$ at $300 \mathrm{~K}$. We find that disorder changes linear scaling to exponential scaling: $\langle N(f)\rangle^{\text {order }}=-4703.4 f+4663.7$ and $\langle N(f)\rangle^{\text {disorder }}=4494.3 \exp (-18.1 f)+48.5$.

value in the ordered state $\left(\mathrm{IPR}_{\max }=6289\right)$ to low values for disordered states $\left(\mathrm{IPR}_{\max }=1652\right.$ and 1961 in 50 and $300 \mathrm{~K}$ cases, respectively), see Figure $6 \mathrm{~b}$. Considering all excitonic states, we find that the effect on IPRs belonging to the red-shift $Q_{\text {y }}$ absorption band is very similar around the peak position $(\lambda$ $-\lambda_{0}>60$ ), see Figure 7, while it is almost absent for exciton states with higher energy, i.e., for $\lambda-\lambda_{0} \approx 0$.

In addition to the IPR, we may quantify (de)localization by considering the exciton density distribution of $\psi_{\max }$, i.e., the exciton state with the highest oscillatory strength, for different molecular conformations. By considering conformations that are taken from a MD trajectory, our tubular states reflect correlations that are responsible for the dynamics of $\mathrm{BChl}$ molecules within this assembly. In this way, we indirectly investigate the role of conformational dynamics on the excitonic behavior. We determine, for each MD configuration, the number of molecules $N(f)$ with an exciton density $p_{i} \geq f$. $p_{\max }$, where $p_{\max }$, defined as the maximum of the exciton density for $\psi_{\max }$ sets the range of the exciton density for each configuration and $f \in[0,1]$ is the corresponding fraction. In particular, $N(f)$ can be seen as a measure for the total area of the exciton domains in $\psi_{\text {max }}$ that correspond to exciton densities that are equal or higher than a fraction $f$. We may now evaluate whether the molecular tube state is significant for $N(f)$ by considering $\langle N(f)\rangle$, where $\langle\cdot\rangle$ denotes the average taken over all considered molecular conformations. Figure 8 shows $\langle N(f)\rangle$ versus $f$ for the ordered assembly at $T=0 \mathrm{~K}$ (one tube state) and for the disordered case, where the averaging is carried out over all snapshots along a $1 \mathrm{~ns}$ MD trajectory at $300 \mathrm{~K}$. Since the standard deviation for the latter case is tiny, we may conclude that the localizing effect of the dynamic disorder as illustrated in Figure 6 is a general feature shared by all tube states. We may exploit these data further to extract an explicit expression for $\langle N(f)\rangle$, i.e., the effective area of the exciton domains ${ }^{57}$ in terms of the threshold fraction $f$. We extracted a linear relation for the ordered case and an exponentially decreasing relation for the disordered case; see Figure 8 .

3.5. Molecular Origins of Exciton Localization. Next, it is important to identify common structural features that correlate with the localization of exciton density. We thus 


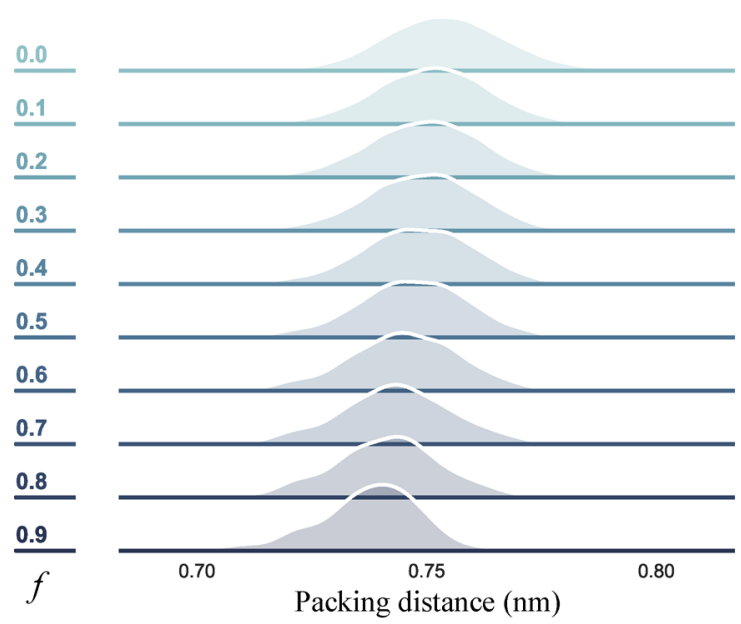

(a)

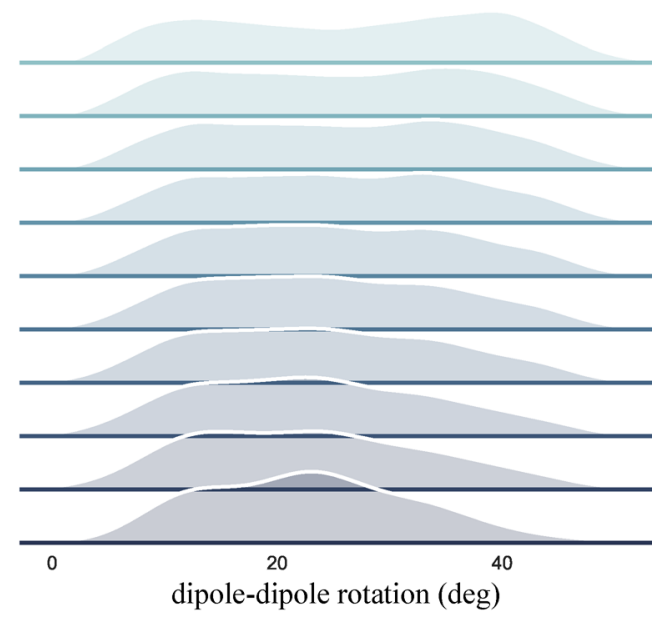

(b)

Figure 9. Structure analysis of molecules at different exciton density levels satisfying $p_{i} \geq f \cdot p_{\max }$ in $\psi_{\max }$ for a $60 \mathrm{~nm}$ long tube with $\delta=49.6^{\circ}$, averaged over a 1 ps MD trajectory at $300 \mathrm{~K}$. (a) Distribution of neighboring $\mathrm{Mg}-\mathrm{Mg}$ distances along the a packing direction and (b) distribution of neighboring dipole-dipole rotation angles along the a packing direction.

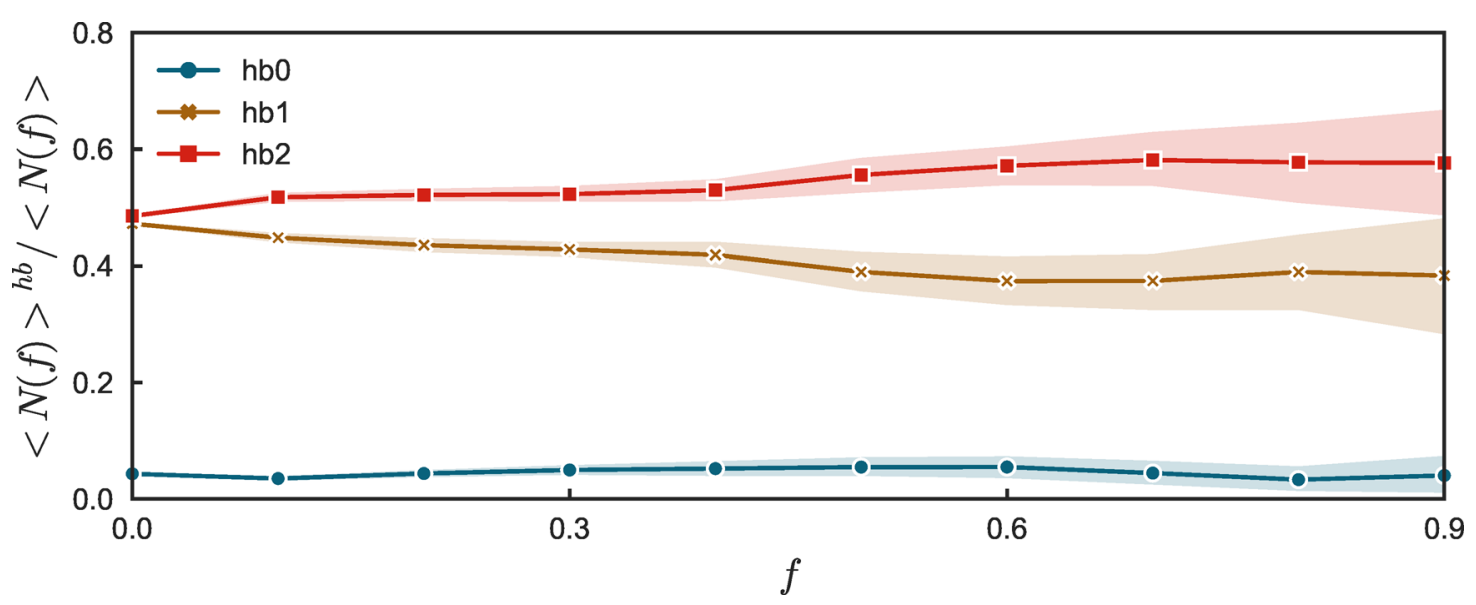

Figure 10. Number of molecules $\langle N(f)\rangle^{h b}$ relative to $\langle N(f)\rangle$ with $0 / 1 / 2$ hydrogen bonds for varying $f$ (horizontal axis), calculated for $\psi_{\text {max }}$ and a 60 $\mathrm{nm}$ tube for $\delta=49.6^{\circ}$ averaged over a $1 \mathrm{ps} \mathrm{MD}$ trajectory simulated at $300 \mathrm{~K}$. Colored bands show the standard derivation.

consider how the dynamic disorder that is present in all tube states along the MD trajectory correlates with high exciton density in $\psi_{\max }$; disorder that was previously evaluated in terms of $\mathrm{Mg}-\mathrm{Mg}$ distances, the rotational angle between neighboring BChl molecules, which is associated with the dipolar interactions, and hydrogen bonding. Following a procedure that is very similar to the one used in the previous section, we determined the distributions of $\mathrm{Mg}-\mathrm{Mg}$ distances $d_{M g-M g}$ and the relative dipolar angles $\alpha_{\text {dipole-dipole }}$ for all molecules in the tubular assembly that satisfy $p_{i} \geq f \cdot p_{\max }$. Combining distributions for all tube states along the trajectory and normalizing, we obtain the two overall distributions shown in Figure 9. From this figure, it is clear that the highest exciton density is associated with the closest $\mathrm{Mg}-\mathrm{Mg}$ separation. The distribution of the rotation angles $\alpha_{\text {dipole-dipole }}$ is clearly bimodal when considered over all molecules in all tubes, i.e., for $f=0$, with peaks around $10^{\circ}$ and $40^{\circ}$. Concentrating only on very high exciton density, $f=0.9$, the overall distribution is almost unimodal, and the broad peak around $40^{\circ}$ has disappeared in favor of a peak at smaller angles. It suggests that molecules that are associated with very high exciton density do not favor large rotations relative to each other. Relative distances and orientations play a role in PDA coupling, so we conclude that both features can be explained in terms of the coupling strength.

Hydrogen bonding was previously identified as an important source of structural heterogeneity. ${ }^{5}$ While a special role of hydrogen bonding in exciton transfer in chlorosomes has been speculated upon, ${ }^{58}$ our current treatment limits itself only to static properties and correlations. Figure 10 shows the ratio of the number of molecules forming 0,1 , and 2 hydrogen bonds versus the fraction $f$ of the exciton density, calculated as an average over all snapshots along a $1 \mathrm{~ns}$ MD trajectory at $T=300$ $\mathrm{K}$; we refer to Figure 8 for information about the number of molecules over which this average is taken. Colored regions show that the standard deviations increase with increasing $f$, which could either be due to variations of these ratios between MD snapshots or the rapidly decreasing number of molecules considered in the averaging procedure. Overall, these data suggest only weak correlation between hydrogen bonding and a high exciton density. Nevertheless, hydrogen bond formation remains a factor in the PDA, albeit only indirectly, through the relation between hydrogen bonding and the relative orientations of induced point dipoles in the molecules.

3.6. Interplay between Dynamics Disorder and Exciton States. Finally, we analyze the exciton states in tube 


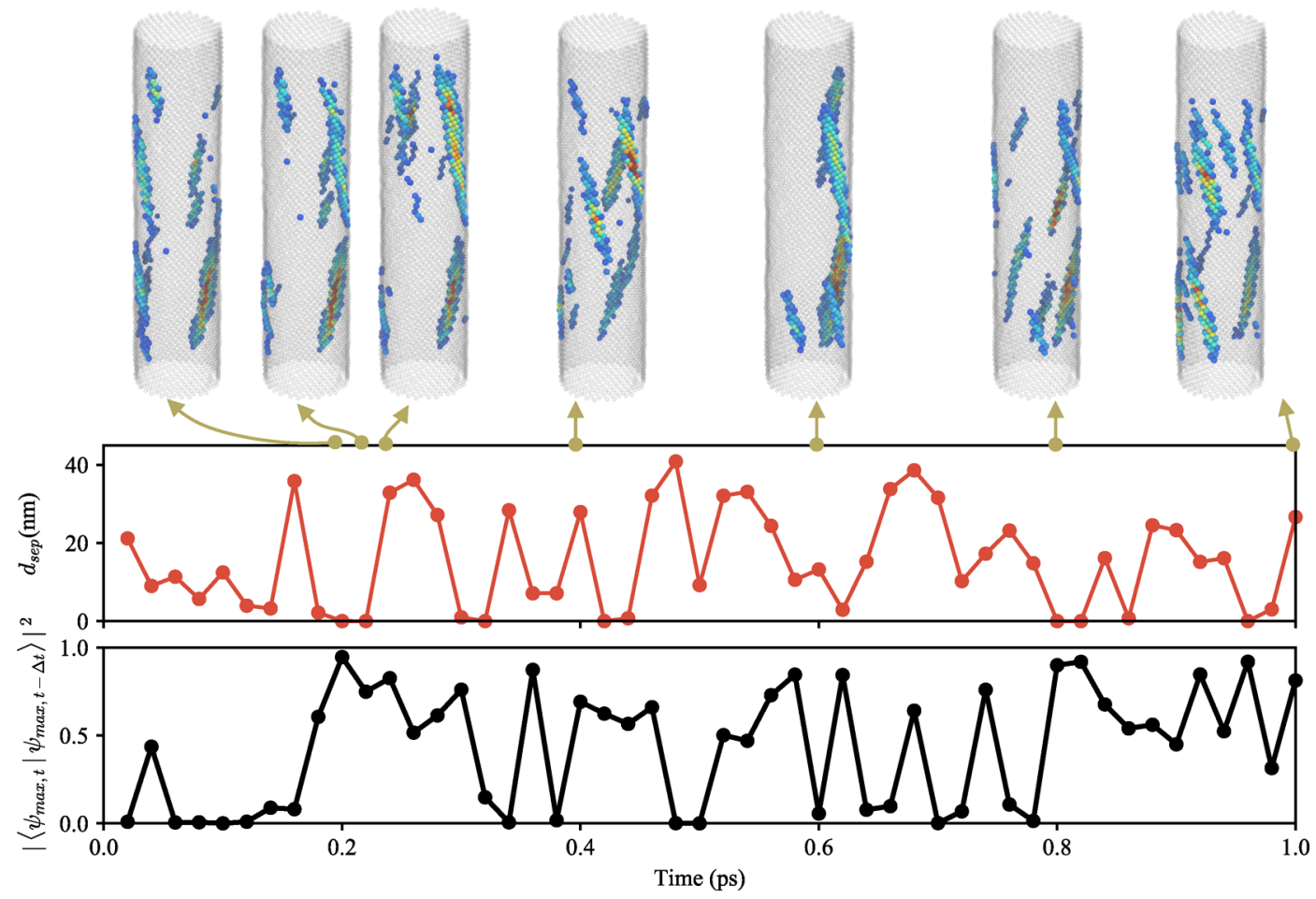

Figure 11. Exciton states $\psi_{\max }$ for different molecular conformations along the $1 \mathrm{ps} \mathrm{MD}$ trajectory for the $\delta=49.6^{\circ}$ tube structure. Top: snapshots of the spatial distribution of $\psi_{\max }$ at different time frames: $0.2,0.22,0.24,0.4,0.6,0.8,1.0 \mathrm{ps}$. Molecules are colored according to the exciton density $p_{i}$. To also visualize the other side of the tube, we made all molecules $i$ with $p_{i}<0.1 p_{\max }$, transparent, where $p_{\max }=0.0066$ is the maximum of the exciton density in all frames. Middle: The distance $d_{\text {sep }}$ between molecules with the largest exciton density in $\psi_{\max }$ in consecutive frames. Bottom: The overlap coefficient, $\left|\left\langle\psi_{\max , t} \mid \psi_{\max , t-\Delta t}\right\rangle\right|^{2}$, between $\psi_{\max }$ in consecutive frames.

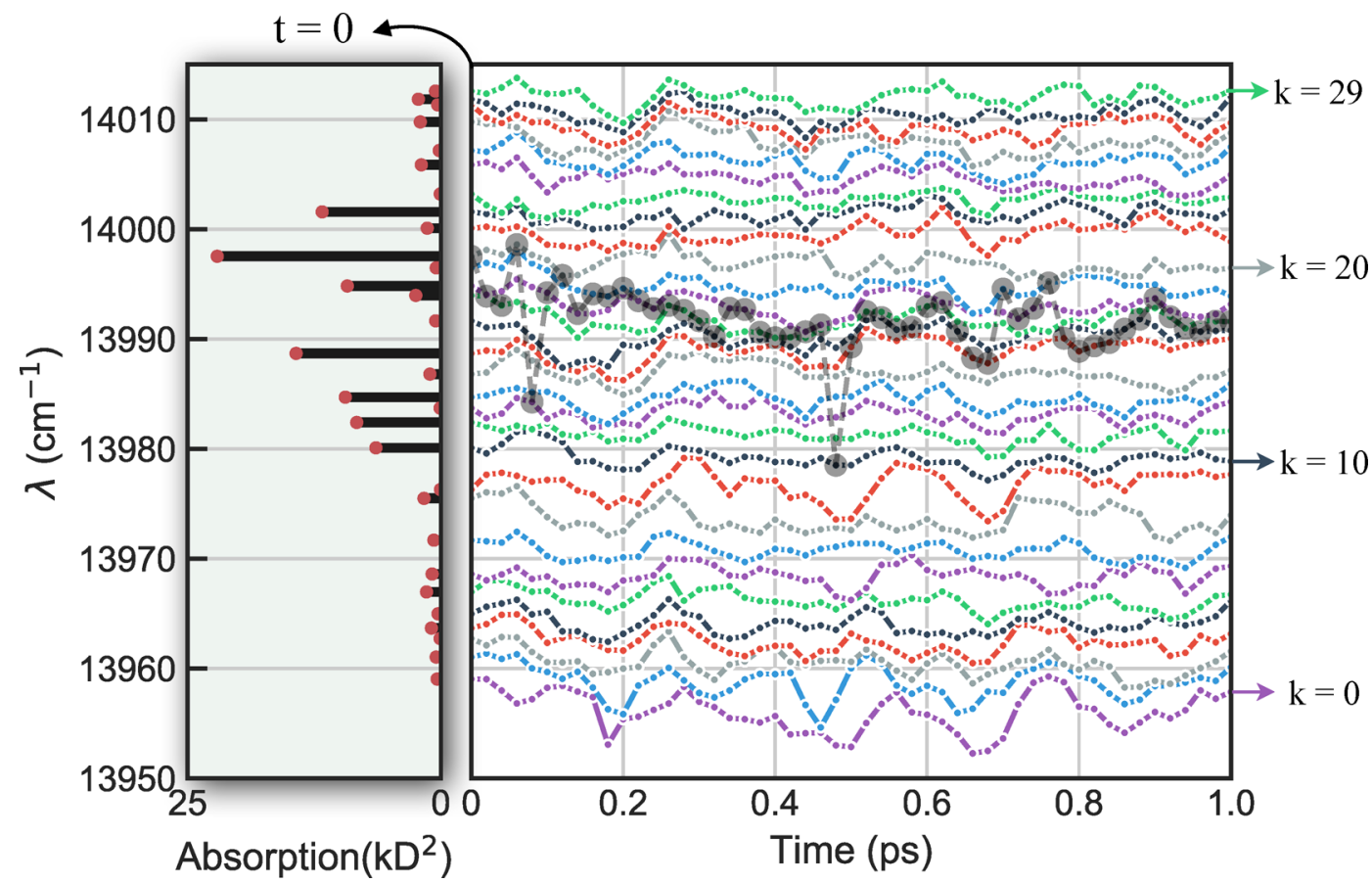

Figure 12. Analysis of stick spectra for the absorption calculated for molecular conformations along a 1 ps MD trajectory for $\delta=49.6^{\circ}$. Left: Stick absorption spectrum calculated for the assembly at $t=0$ ps. Right: The values of the 30 lowest energies associated with 30 eigenstates of the Frenkel Hamiltonian. Eigenstates and their energies are calculated for snapshots along the 1 ps MD trajectory. Energies for individual snapshots are ranked from low to high, starting from $k=m-1=0$, see Method section, and colored according to their rank. The energy associated with the largest oscillator strength $\psi_{\max }$ for each snapshot is denoted by a gray sphere.

assemblies that are extracted from different frames along MD trajectories to evaluate their response to dynamic disorder that is induced by thermal motion. Exciton states are analyzed both in real space and in Hilbert space, and we extended our 


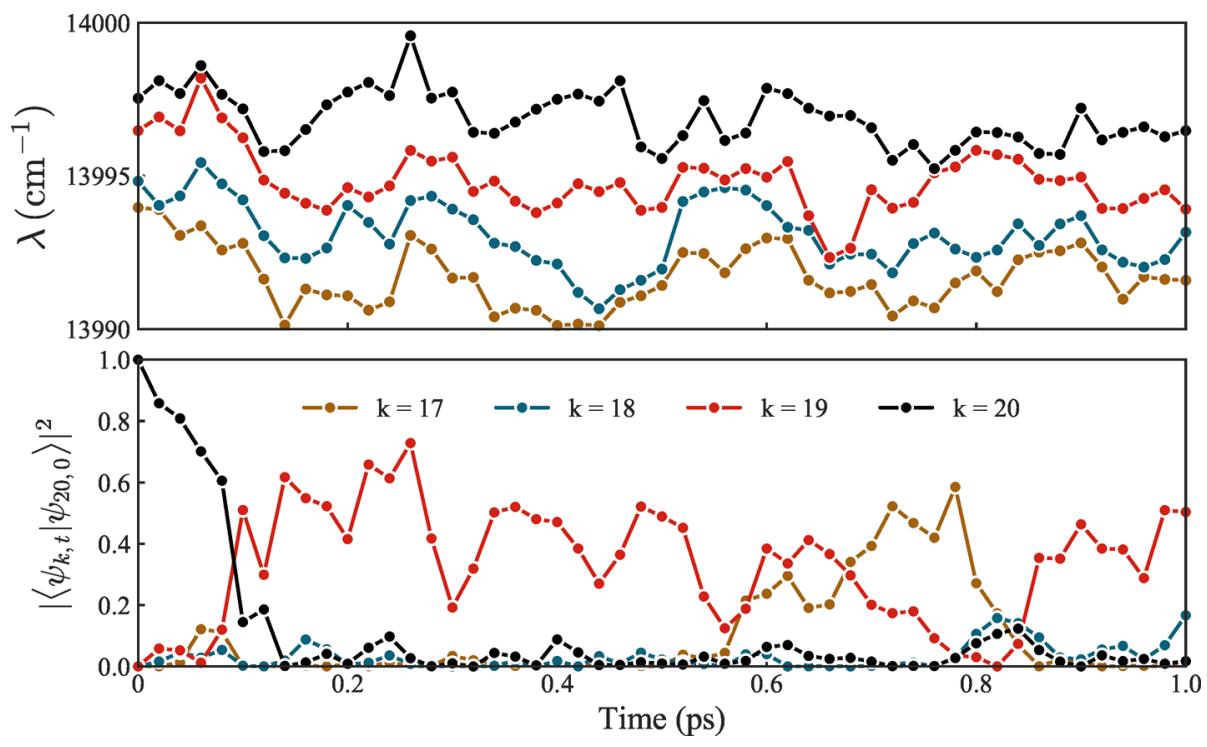

Figure 13. Overlap coefficient, $\left|\left\langle\psi_{k, t} \mid \psi_{20,0}\right\rangle\right|^{2}$, between selected states $\psi_{k, t}$ and the absorption state $\psi_{20,0}$ at $t=0$ ps, along a 1 ps MD trajectory for $\delta=$ $49.6^{\circ}$. In line with this definition, the initial overlap coefficient $=1.0$ for $k=20$ state. States $k=17,18,19$, and 20 are labeled by different colors.

calculations to three concentric tubes to evaluate intertube correlation and delocalization. As mentioned, our current investigations provide no direct information on the role of temperature $^{14}$ or vibrational coherence ${ }^{13,55}$ in exciton migration, since we do not include a propagator at the quantum level. Representing nuclear responses to excitation in a MD force field is currently an open issue ${ }^{59}$ and remains challenging, ${ }^{60}$ albeit that electronic ground state nuclear dynamics is considered a justified choice for these systems. ${ }^{36}$

Exciton migration in chlorosomes is a fast process, ${ }^{3,23,61}$ with an initial energy flow identified to occur in the first $200 \mathrm{fs}$ after excitation. ${ }^{13,14,55,56}$ The two distinct low frequency modes recently identified by ultrafast $2 \mathrm{D}$ spectroscopy for chlorosomes at $80 \mathrm{~K}$ relate to coherent beatings with a period of 230/366 fs on a characteristic time scale of ps. ${ }^{55}$ We have thus performed a 1 ps MD simulation and analyzed the resulting trajectory with a time resolution of $20 \mathrm{fs}$. As the time interval ( $20 \mathrm{fs}$ ) is small, the lattice site (based on $\mathrm{Mg}$ atom position) variation between neighboring frames is small, with

$$
\operatorname{RMSD}_{\text {lattice }}=\sqrt{\frac{1}{N} \sum_{i}^{N}\left(x_{i}(t)-x_{i}(t-\Delta t)\right)^{2}}
$$

being less than $0.01 \mathrm{~nm}$ when calculated over all $N$ positions of the $\mathrm{Mg}$ atoms $x_{i}$, for both the single tube and concentric tube trajectories.

3.6.1. Single Tube. We analyze single tube aggregates by projecting the exciton distribution for the largest oscillator strength $\psi_{\max }$ onto the structure using color coding, see Figure 11 and Movie S1. We have also plotted the separation distance $d_{\text {sep }}$ between molecules that feature the largest exciton density $p_{\max }$ of $\psi_{\max }$ in consecutive frames. The comparable RMSD lattice $=0.148 \pm 0.018 \mathrm{~nm}$ and $d_{\text {sep }}$ behavior for the longer $500 \mathrm{ps}$ trajectory, see Figure S11 and Movie S2, show that the short trajectory is representative. While the fluctuating character of $d_{\text {sep }}$ suggests equivalent changes to the corresponding exciton distributions, these distributions can be quite similar; see Figure 11. Conversely, changes in the exciton distribution in shape, (dis)appearance and shifts along the tube are taking place during the whole 1 ps trajectory, even when the local measure $d_{\text {sep }} \approx 0$. Analyzing the variation of the absorption state $\psi_{\max }$ for different molecular conformations along the MD trajectory using a global measure, i.e., the overlap coefficient $\left|\left\langle\psi_{\text {max }, t} \mid \psi_{\text {max }, t-\Delta t}\right\rangle\right|^{2}$ of $\psi_{\text {max }}$ in consecutive frames, see Figure 11 (bottom), shows that this absorption state indeed significantly varies with the underlying molecular conformations.

The fluctuating character of the local measure for the response of the exciton distribution to the nuclear dynamics, $d_{\text {sep }}$, suggests that the selection of $\psi_{\text {max }}$ is quite sensitive to small changes in the underlying molecular structure. This agrees well with the earlier suggestion that very fast energy transfer is facilitated by energy crossing within the manifold of exciton states with very similar energies. ${ }^{13}$ Additional analysis of the 30 lowest eigenvalues of our Frenkel Hamiltonians, see Figure 12, enables us to study this suggestion. We find that energies fluctuate as a result of dynamic disorder in the underlying molecular assembly that is reflected in the Frenkel Hamiltonian, but the amplitudes of these fluctuations are small in an absolute sense. Note that the time scale in Figure 12 is added to provide insight in the evolution of the tube structure along the $\mathrm{MD}$ trajectory, whereas our analysis of exciton states remains essentially static. We will nevertheless label eigenstates according to $\mathrm{MD}$ time for ease of discussion. Following the eigenstate with the largest contribution to absorption, $\psi_{\max }$, we find that its position shifts within a tiny band of $20 \mathrm{~cm}^{-1}$. Moreover, the energies of all 30 eigenstates lie within a narrow $60 \mathrm{~cm}^{-1}$ band for all considered molecular conformations. This proximity of energy levels is an indication that nonadiabatic energy transfer is an option, provided that states also have a spatial overlap.

Our choice to rank eigenstates according to their eigenvalues obscures potential crossings, but we note that also the eigenstates change with the considered tube state. The crossing of energy levels can however be anticipated from several anticorrelated kinks that are present in Figure 12. Introducing additional overlap coefficients, $\left|\left\langle\psi_{k, t} \mid \psi_{20,0}\right\rangle\right|^{2}$, between $\psi_{k, t}$ for different $k$ and the initial absorption state $\psi_{20,0}$ further quantifies this phenomenon. Focusing on the overlap coefficients for $k=$ 
(a)

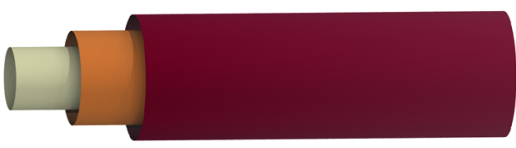

$\bigcirc$ tube $1 \bigcirc$ tube $2 \bigcirc$ tube 3 (c)

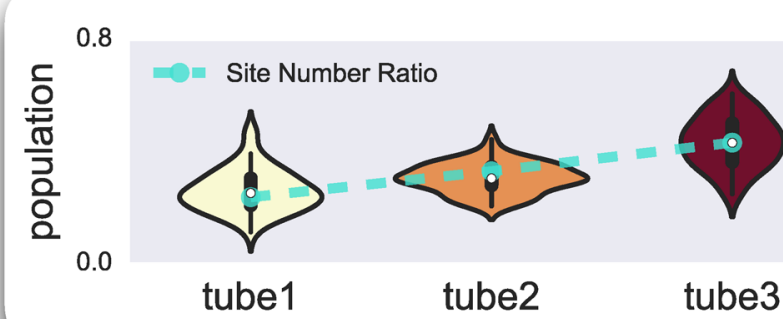

(b)
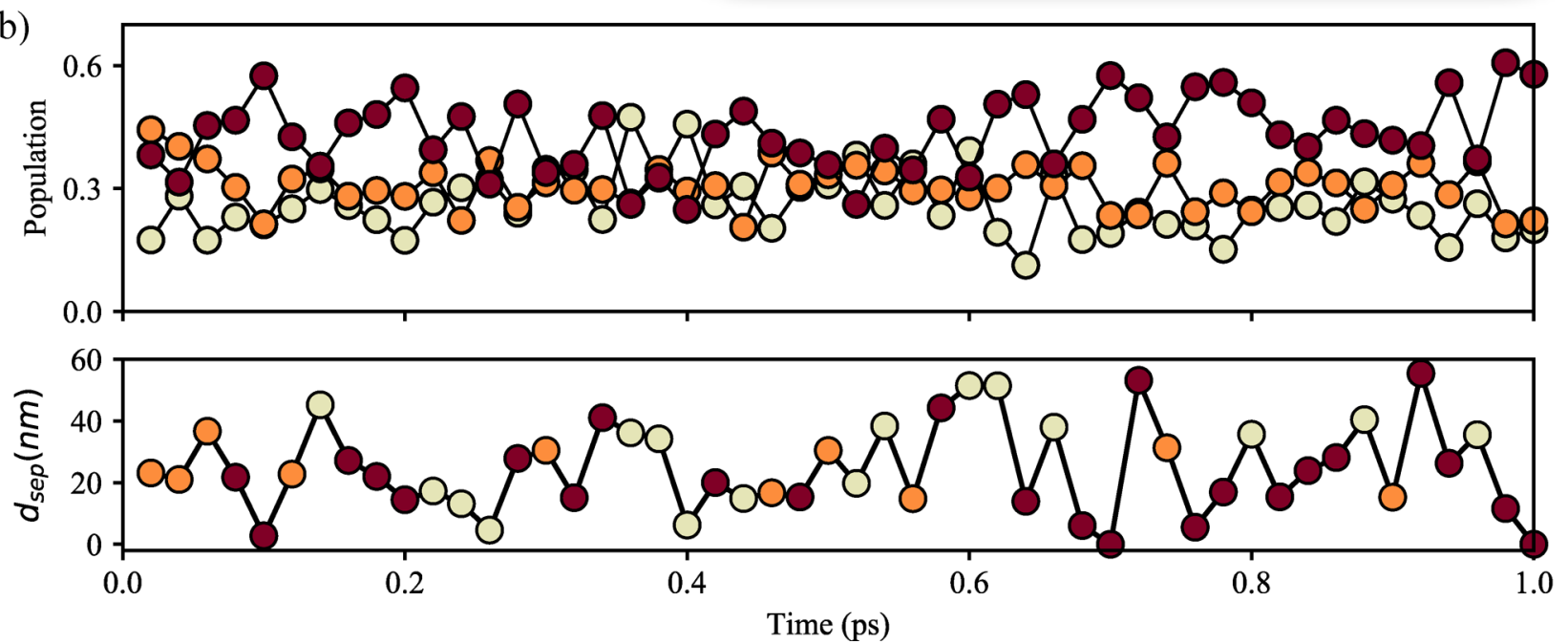

Figure 14. Analysis of the excitons states $\psi_{\max }$ in concentric 3-tube structures. (a) Schematic illustration of concentric tube geometry, with radii satisfying tube $3>$ tube $2>$ tube 1 . Colors are used to distinguish between different tubes. (b) Top: the exciton density population in different tubes, denoted by tube color, along a 1 ps trajectory. Bottom: $d_{s e p}$, the distance between molecules associated with the largest exciton density in $\psi_{\text {max }}$ in consecutive frames. Color specify in which tube the molecule with the highest exciton density is situated. (c) The distribution of exciton density population of $\psi_{\max }$ over all frames in the trajectory. The average (white point) agrees well with the trend of the normalized number of molecules (site number ratio, dashed line) in each tube.

17, 19, and 20, see Figure 13, we find that the initial eigenstate for $k=20$ becomes orthogonal to $\psi_{20, t}$ within $200 \mathrm{fs}$. As time proceeds, the initial state $\psi_{20,0}$ shows significant overlap with other eigenstates with a different energy rank, $\psi_{19, t}$ and later $\psi_{17, t}$. These results indicate that energy crossing can indeed be facilitated by dynamic disorder.

Concentrating on the projected $\psi_{\max }$ itself, domains can be seen always aligned at an angle with the long cylinder axis, somewhere between the a packing direction and the hydrogenbonding direction, as these two directions accommodate the strongest coupled pairs. In particular, see Figure 11 (top), this preferential angle is determined by contributions of all helical families and modulated by factors such as dynamic disorder and the electronic coupling model considered. Our findings reveal the difficulty of relating optical responses directly to properties of the underlying molecular lattices.

3.6.2. Three-Layered Tubular Assembly. In chlorosomes, energy produced by a photon exciting the inner tube needs to migrate within $10 \mathrm{ps}$ (the average annihilation time) ${ }^{62}$ to the outer tube to reach the baseplate and subsequently the reaction center. Thus, we performed an analysis for a dense 1 ps MD trajectory ( $20 \mathrm{fs}$ per frame, $300 \mathrm{~K}$ ) of a concentric three-tube complex (Figure 14a) to explore the relation between dynamic disorder and excitonic properties, and to propose a mechanism that leads to accumulation of excitonic energy in the outer layers. The concentric structure is composed of three prepacked tubes with radii $R=5.2,7.3$, and $9.5 \mathrm{~nm}$, separated by $2.1 \mathrm{~nm}$, as observed in experiments. ${ }^{2}$ Before collecting the trajectory, the structure was well equilibrated by the standard procedure, see Methods, and found to be stable.

Since concentric tubes in a multitube structure can be assembled in two distinct relative orientations, either parallel or antiparallel to each other, we also considered a two-layered assembly and calculated spectral properties for these two different options. Although the stick spectra display tiny differences, the calculated absorption, $\mathrm{LD}$ and $\mathrm{CD}$ spectra are equivalent after broadening. This finding shows that the current experimental resolution is insufficient to distinguish relative tube orientations. For this reason, we further concentrate on a situation where all tubes are parallel to each other.

Key features identified for a single tube are also found for the concentric tubes: extremely low structure variation (RMSD lattice $=0.009 \pm 0.00003 \mathrm{~nm})$ and substantial variation of the separation distance $d_{\text {sep }}$ (Figure 14b). Again, $d_{\text {sep }}$ is 3 orders of

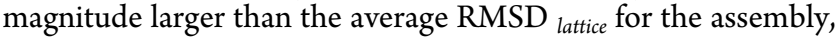
and the average value of $24.7 \mathrm{~nm}$ is about 10 times the gap distance between adjacent layers, suggesting that the position of maximum exciton density in $\psi_{\max }$ may well migrate from tube to tube. From the data shown in Figure 14b, we see that this is indeed the case. Since visualization of the exciton distribution for $\psi_{\max }$ in the tubular complex is less straightforward than for a single tube, we determined the fractional population of this state in each of the three tubes, see Figure 14b. It shows that $\psi_{\text {max }}$ is indeed delocalized over all three tubes and that the 
exciton fraction in each of the tubes fluctuates around the average value with a considerable amplitude. In particular, the fraction present in the outer tube (tube 3 ) is seen to be the largest for most of the molecular conformations along the MD trajectory. Plotting the distribution of the exciton fractional population in each tube separately, see Figure 14c, shows a clear radial dependence, with the population averages (0.26:0.31:0.43) closely following the molecular ratio (0.24:0.33:0.43) for each of the three tubes. We thus conclude that the distribution of excitonic energy over each of the tubes does not reflect variations in disorder but directly relates to the number of molecules that is needed to constitute the tube. Since this number increases with increased tube radius $R$, exciton energy will automatically concentrate in the outer part of the tubular complex. The conclusions obtained from the short and dense trajectory ( $1 \mathrm{ps)}$ also apply to the long and dilute trajectory $(500 \mathrm{ps})$, see the structure and exciton state analysis shown in the Figure S12. To further investigate the nature of this coupling, we considered a special case, i.e., the electronic couplings $J_{i k}=0$ when $i$ and $k$ sites belong to tube 1 or tube 3 , and $J_{i k} \neq 0$ when one of the sites is located in tube 2 . For this $J$, we find that most of exciton density of $\psi_{\max }$ is confined to tube 2 , as expected. In tubes 1 and 3 , however, a tiny signal can be observed, indicating that intertube coupling is weak but present, even in the case where internal electronic coupling is suppressed.

\section{CONCLUSIONS}

Understanding how structural properties of protein-free BChl aggregates like chlorosomes translate into uniquely efficient and robust transfer of excitation energy is a significant but unresolved scientific challenge. In the present work, we have developed a protocol that combines experimental information from NMR, cryo-EM and optical spectroscopy to constitute a unique model of these biological assemblies. Our model is unique in allowing for realistic disorder and for reproducing all available characterization data simultaneously, including typical features of the absorption, linear dichroism (LD), and circular dichroism (CD) spectra.

The developed protocol is general, and it can be applied for other helical tube forming molecules. First, our extended geometrical analysis relates the axial repeat distance $d$ of experimentally obtained layer lines ${ }^{2}$ to chiral angles $\delta \in\left[0^{\circ}\right.$, $180^{\circ}$ ]. Consequently, we employ this set of $\delta$, which is a key structural parameter together with the previously determined syn-anti dimer unit cell ${ }^{2}$ and Bravais lattice parameters $(a, b$, $\gamma),{ }^{5}$ to build tubes for MD simulation of a realistic size, i.e., $l=$ $120 \mathrm{~nm}$ length and $R=7.5 \mathrm{~nm}$ radius. After confirming stability at $T=300 \mathrm{~K}$, a Frenkel exciton Hamiltonian is used to calculate optical spectra of ordered and disordered tubes for a direct comparison to measured spectral types. In this study, we have adopted the point-dipole approximation for the electronic coupling calculation, but other descriptions are optional. Our calculations show that $\mathrm{CD}$ spectra types change several times along the $\delta$-range, for instance from a type II for $\delta=42.3^{\circ}$ to a mixed-type for $\delta=27.6^{\circ}$. This finding explains the previously mentioned "uncontrolled" variation of CD signal type, ${ }^{20,42}$ e.g., between type II and mixed-type, which is likely due to small modulations of the overall tube chirality due to compositional heterogeneity or growth condition rather than insufficient tube length or different curving directions in a mixture of tubes. ${ }^{2,6,20}$

Next, we clarified the intricate role of disorder on exciton states, with a focus on the state with the largest oscillator strength. We find disorder to induce localization of exciton states, i.e., concentration of exciton density in a spatially confined region, to a degree that is consistent with experimental observations. ${ }^{23}$ We quantify this effect by considering distribution profiles of exciton states, their inverse participation ratios and corresponding exciton distributions mapped onto the tubular structure. These mapped distributions for the first time visualize the insight derived from $2 \mathrm{D}$ spectroscopy, which described chlorosomes as composed of weakly coupled coherent domains. ${ }^{13}$ The observation that relatively small variations of the underlying molecular structure translate into significant changes in the exciton structure, giving rise to crossings between eigenstates with energies that are part of a tiny band, is in good agreement with earlier suggestion that the presence of many states of comparable energy enhances intratube migration of excitons. ${ }^{13}$ Focusing on a concentric tube case, we find the ratio between exciton population in different tubes, when averaged over the MD trajectory, agrees well with their molar ratio, suggesting the driving force for the migration of exciton energy from inside to outside that is simply the fact that larger tubes contain more molecules, since $N \propto R^{2}$. We plan to investigate the detailed role of static and dynamic disorder on actual exciton migration in future work.

\section{ASSOCIATED CONTENT}

\section{Supporting Information}

The Supporting Information is available free of charge on the ACS Publications website at DOI: 10.1021/acs.jpcc.9b03913.

Theoretical prediction of axial repeat distance $d$; EM mimics for different $\delta$ tube structures; lattice structural properties of tube structures; optical spectral plots for tube structures of different chiralities, lengths, and simulation temperatures; head-head rotation distribution for different $\delta$ tube structures; spatial visualization of the exciton states $\psi_{\max }$ in a $120 \mathrm{~nm}$ long tube of different simulation temperatures; and structural and exciton state variation along a 500 ps $\mathrm{MD}$ trajectory for a single tube and concentric 3-tube (PDF)

Spatial visualization of exciton states $\psi_{\max }$ for different molecular conformations of the $\delta=49.6^{\circ}$ tube along a 1 ps MD trajectory (Movie S1) (MPG)

Spatial visualization of exciton states $\psi_{\max }$ for different molecular conformations of the $\delta=49.6^{\circ}$ tube along a 500 ps MD trajectory (Movie S2) (MPG)

\section{AUTHOR INFORMATION}

\section{Corresponding Authors}

*(X.L.) E-mail: x.li@lic.leidenuniv.nl.

*(G.J.A.S.) E-mail: a.sevink@chem.leidenuniv.nl.

ORCID

Xinmeng Li: 0000-0002-6863-6078

Francesco Buda: 0000-0002-7157-7654

G. J. Agur. Sevink: 0000-0001-8005-0697

Notes

The authors declare no competing financial interest.

\section{ACKNOWLEDGMENTS}

The authors thank Lina Kan for performing reference simulations of mixed BChl $c-d$ systems. The authors acknowledge the helpful discussion with Dr. T. L. C. Jansen about the electronic coupling calculation. The use of supercomputer 
facilities was sponsored by NWO Physical Sciences, with financial support from The Netherlands Organization for Scientific Research (NWO).

\section{REFERENCES}

(1) Oostergetel, G. T.; van Amerongen, H.; Boekema, E. J. The Chlorosome: a Prototype for Efficient Light Harvesting in Photosynthesis. Photosynth. Res. 2010, 104, 245-255.

(2) Ganapathy, S.; Oostergetel, G. T.; Wawrzyniak, P. K.; Reus, M.; Gomez Maqueo Chew, A.; Buda, F.; Boekema, E. J.; Bryant, D. A.; Holzwarth, A. R.; de Groot, H. J. M. Alternating Syn-Anti Bacteriochlorophylls Form Concentric Helical Nanotubes in Chlorosomes. Proc. Natl. Acad. Sci. U. S. A. 2009, 106, 8525-8530.

(3) Orf, G. S.; Blankenship, R. E. Chlorosome Antenna Complexes from Green Photosynthetic Bacteria. Photosynth. Res. 2013, 116, 315331.

(4) Tian, Y.; Camacho, R.; Thomsson, D.; Reus, M.; Holzwarth, A. R.; Scheblykin, I. G. Organization of Bacteriochlorophylls in Individual Chlorosomes from Chlorobaculum tepidum Studied by 2-Dimensional Polarization Fluorescence Microscopy. J. Am. Chem. Soc. 2011, 133, 17192-17199.

(5) Li, X.; Buda, F.; de Groot, H. J. M.; Sevink, G. J. A. Contrasting Modes of SelfAssembly and Hydrogen-Bonding Heterogeneity in Chlorosomes of Chlorobaculum tepidum. J. Phys. Chem. C 2018, 122, 14877-14888.

(6) Prokhorenko, V. I.; Steensgaard, D. B.; Holzwarth, A. R. Exciton Theory for Supramolecular Chlorosomal Aggregates: 1. Aggregate Size Dependence of the Linear Spectra. Biophys. J. 2003, 85, 3173-3186.

(7) Günther, L. M.; Jendrny, M.; Bloemsma, E. A.; Tank, M.; Oostergetel, G. T.; Bryant, D. A.; Knoester, J.; Köhler, J. Structure of Light-Harvesting Aggregates in Individual Chlorosomes. J. Phys. Chem. B 2016, 120, 5367-5376.

(8) Saikin, S. K.; Eisfeld, A.; Valleau, S.; Aspuru-Guzik, A. Photonics Meets Excitonics: Natural and Artificial Molecular Aggregates. Nanophotonics 2013, 2, 21-38.

(9) Fassioli, F.; Dinshaw, R.; Arpin, P. C.; Scholes, G. D. Photosynthetic Light Harvesting: Excitons and Coherence. J. R. Soc., Interface 2014, 11, 20130901.

(10) Didraga, C.; Klugkist, J. A.; Knoester, J. Optical Properties of Helical Cylindrical Molecular Aggregates: the Homogeneous Limit. J. Phys. Chem. B 2002, 106, 11474-11486.

(11) Linnanto, J. M.; Korppi-Tommola, J. E. I. Exciton Description of Chlorosome to Baseplate Excitation Energy Transfer in Filamentous Anoxygenic Phototrophs and Green Sulfur Bacteria. J. Phys. Chem. B 2013, 117, 11144-11161.

(12) Somoza Marquez, A.; Chen, L.; Sun, K.; Zhao, Y. Probing Ultrafast Excitation Energy Transfer of the Chlorosome with ExcitonPhonon Variational Dynamics. Phys. Chem. Chem. Phys. 2016, 18, 20298-20311.

(13) Dostál, J.; Mančal, T.; Augulis, R.-n.; Vácha, F.; Pšenčík, J.; Zigmantas, D. TwoDimensional Electronic Spectroscopy Reveals Ultrafast Energy Diffusion in Chlorosomes. J. Am. Chem. Soc. 2012, 134, 11611-11617.

(14) Jun, S.; Yang, C.; Kim, T. W.; Isaji, M.; Tamiaki, H.; Ihee, H.; Kim, J. Role of Thermal Rxcitation in Ultrafast Energy Transfer in Chlorosomes Revealed by Two-Dimensional Electronic Spectroscopy. Phys. Chem. Chem. Phys. 2015, 17, 17872-17879.

(15) Bussi, G.; Donadio, D.; Parrinello, M. Canonical Sampling through Velocity Rescaling. J. Chem. Phys. 2007, 126, No. 014101.

(16) Malý, P.; van Grondelle, R. Interplay of Disorder and Delocalization in Photosynthetic Light Harvesting. Curr. Opin. Chem. Biol. 2018, 47, 1-6.

(17) Agranovich, V. M.; Galanin, M. D. Electronic Excitation Energy Transfer in Condensed Matter ; North-Holland: Amsterdam, 1982.

(18) Kenny, E. P.; Kassal, I. Benchmarking Calculations of Excitonic Couplings between Bacteriochlorophylls. J. Phys. Chem. B 2016, 120, $25-32$.
(19) Fujita, T.; Brookes, J. C.; Saikin, S. K.; Aspuru-Guzik, A. Memory-Assisted Exciton Diffusion in the Chlorosome Light-Harvesting Antenna of Green Sulfur Bacteria. J. Phys. Chem. Lett. 2012, 3, 2357-2361.

(20) Tang, J. K.-H.; Saikin, S. K.; Pingali, S. V.; Enriquez, M. M.; Huh, J.; Frank, H. A.; Urban, V. S.; Aspuru-Guzik, A. Temperature and Carbon Assimilation Regulate the Chlorosome Biogenesis in Green Sulfur Bacteria. Biophys. J. 2013, 105, 1346-1356.

(21) Huh, J.; Saikin, S. K.; Brookes, J. C.; Valleau, S.; Fujita, T.; Aspuru-Guzik, A. Atomistic Study of Energy Funneling in the LightHarvesting Complex of Green Sulfur Bacteria. J. Am. Chem. Soc. 2014, $136,2048-2057$.

(22) Oviedo, M. B.; Sánchez, C. G. Transition Dipole Moments of the $\mathrm{Q}_{\mathrm{y}}$ Band in Photosynthetic Pigments. J. Phys. Chem. A 2011, 115, $12280-12285$.

(23) Prokhorenko, V. I.; Steensgaard, D. B.; Holzwarth, A. R. Exciton Dynamics in the Chlorosomal Antennae of the Green Bacteria Chloroflexus aurantiacus and Chlorobium tepidum. Biophys. J. 2000, $79,2105-2120$.

(24) Kriete, B.; Bondarenko, A. S.; Jumde, V. R.; Franken, L. E.; Minnaard, A. J.; Jansen, T. L. C.; Knoester, J.; Pshenichnikov, M. S. Steering Self-Assembly of Amphiphilic Molecular Nanostructures via Halogen Exchange. J. Phys. Chem. Lett. 2017, 8, 2895-2901.

(25) Bloemsma, E. A.; Vlaming, S. M.; Malyshev, V. A.; Knoester, J. Signature of Anomalous Exciton Localization in the Optical Response of Self-Assembled Organic Nanotubes. Phys. Rev. Lett. 2015, 114, 156804.

(26) Molina, R. A.; Benito-Matías, E.; Somoza, A. D.; Chen, L.; Zhao, Y. Superradiance at the Localization-Delocalization Crossover in Tubular Chlorosomes. Phys. Rev. E: Stat. Phys., Plasmas, Fluids, Relat. Interdiscip. Top. 2016, 93, No. 022414.

(27) Pearlstein, R. M. In Chlorophylls; Scheer, H., Ed.; CRC Press: New York, 1991; pp 1047-1078.

(28) Chenu, A.; Scholes, G. D. Coherence in Energy Transfer and Photosynthesis. Annu. Rev. Phys. Chem. 2015, 66, 69-96.

(29) González, L.; Escudero, D.; Serrano-Andrés, L. Progress and Challenges in the Calculation of Electronic Excited States. ChemPhysChem 2012, 13, 28-51.

(30) Cupellini, L.; Jurinovich, S.; Campetella, M.; Caprasecca, S.; Guido, C. A.; Kelly, S. M.; Gardiner, A. T.; Cogdell, R.; Mennucci, B. $\mathrm{An} \mathrm{Ab}$ Initio Description of the Excitonic Properties of LH2 and Their Temperature Dependence. J. Phys. Chem. B 2016, 120, 11348-11359.

(31) Adolphs, J.; Müh, F.; Madjet, M. E.-A.; Renger, T. Calculation of Pigment Transition Energies in the FMO Protein. Photosynth. Res. 2008, 95, 197-209.

(32) Rivera, E.; Montemayor, D.; Masia, M.; Coker, D. F. Influence of Site-Dependent Pigment-Protein Interactions on Excitation Energy Transfer in Photosynthetic Light Harvesting. J. Phys. Chem. B 2013, $117,5510-5521$.

(33) Rancova, O.; Abramavicius, D. Static and Dynamic Disorder in Bacterial LightHarvesting Complex LH2: A 2DES Simulation Study. J. Phys. Chem. B 2014, 118, 7533-7540.

(34) Matysik, J.; Alia; Gast, P.; van Gorkom, H. J.; Hoff, A. J.; de Groot, H. J. M. Photochemically Induced Nuclear Spin Polarization in Reaction Centers of Photosystem II Observed by 13C-solid-state NMR Reveals a Strongly Asymmetric Electronic Structure of the P680(.+) Primary Donor Chlorophyll. Proc. Natl. Acad. Sci. U. S. A. 2000, 97, 9865-9870.

(35) Caffarri, S.; Tibiletti, T.; Jennings, R.; Santabarbara, S. A Comparison between Plant Photosystem I and Photosystem II Architecture and Functioning. Curr. Protein Pept. Sci. 2014, 15, 296-331.

(36) Bouvier, B.; Dognon, J.-P.; Lavery, R.; Markovitsi, D.; Millié, P.; Onidas, D.; Za- krzewska, K. Influence of Conformational Dynamics on the Exciton States of DNA Oligomers. J. Phys. Chem. B 2003, 107, 13512-13522.

(37) Fujita, T.; Huh, J.; Saikin, S. K.; Brookes, J. C.; Aspuru-Guzik, A. Theoretical Characterization of Excitation Energy Transfer in 
Chlorosome Light-Harvesting Antennae from Green Sulfur Bacteria. Photosynth. Res. 2014, 120, 273-289.

(38) Sachse, C. Single-Particle Based Helical Reconstruction-How to Make the Most of Real and Fourier Space. AIMS Biophys. 2015, 2, 219-244.

(39) van Dorssen, R. J.; Vasmel, H.; Amesz, J. Pigment Organization and Energy Transfer in the Green Photosynthetic Bacterium Chloroflexus Aurantiacus. Photosynth. Res. 1986, 9, 33-45.

(40) van Amerongen, H.; Vasmel, H.; van Grondelle, R. Linear Dichroism of Chlorosomes from Chloroflexus Aurantiacus in Compressed Gels and Electric Fields. Biophys. J. 1988, 54, 65-76.

(41) Griebenow, K.; Holzwarth, A. R. Pigment Organization and Energy Transfer in Green Bacteria 1. Isolation of Native Chlorosomes Free of Bound Bacteriochlorophyll $a$ from Chloroflexus Aurantiacus by Gel-Electrophoretic Filtration. Biochim. Biophys. Acta, Bioenerg. 1989, 973, 235-240.

(42) Griebenow, K.; Holzwarth, A. R.; van Mourik, F.; van Grondelle, R. Pigment Organiza- tion and Energy Transfer in Green Bacteria. 2. Circular and Linear Dichroism Spectra of Protein-Containing and Protein-Free Chlorosomes Isolated from Chloroflexus Aurantiacus Strain Ok-70-fl. Biochim. Biophys. Acta, Bioenerg. 1991, 1058, 194-202.

(43) Lehmann, R. P.; Brunisholz, R. A.; Zuber, H. Giant Circular Dichroism of Chlorosomes from Chloroflexus Aurantiacus Treated with 1-Hexanol and Proteolytic Enzymes. Photosynth. Res. 1994, 41, $165-173$.

(44) Frese, R.; Oberheide, U.; van Stokkum, I.; van Grondelle, R.; Foidl, M.; Oelze, J.; van Amerongen, H. The Organization of Bacteriochlorophyll $c$ in Chlorosomes from Chloroflexus Aurantiacus and the Structural Role of Carotenoids and Protein. Photosynth. Res. 1997, 54, 115-126.

(45) Tang, K.-H.; Zhu, L.; Urban, V. S.; Collins, A. M.; Biswas, P.; Blankenship, R. E. Temperature and Ionic Strength Effects on the Chlorosome Light-Harvesting Antenna Complex. Langmuir 2011, 27, $4816-4828$.

(46) Furumaki, S.; Vacha, F.; Habuchi, S.; Tsukatani, Y.; Bryant, D. A.; Vacha, M. Absorption Linear Dichroism Measured Directly on a Single Light-Harvesting System: The Role of Disorder in Chlorosomes of Green Photosynthetic Bacteria. J. Am. Chem. Soc. 2011, 133, 67036710.

(47) Garab, G.; van Amerongen, H. Linear Dichroism and Circular Dichroism in Photosynthesis Research. Photosynth. Res. 2009, 101, 135-146.

(48) Betti, J. A.; Blankenship, R. E.; Natarajan, L. V.; Dickinson, L. C.; Fuller, R. C. Antenna Organization and Evidence for the Function of a New Antenna Pigment Species in the Green Photosynthetic Bacterium Chloroflexus Aurantiacus. Biochim. Biophys. Acta, Bioenerg. 1982, 680, 194-201.

(49) Eisele, D. M.; Cone, C. W.; Bloemsma, E. A.; Vlaming, S. M.; van der Kwaak, C. G. F.; Silbey, R. J.; Bawendi, M. G.; Knoester, J.; Rabe, J. P.; Vanden Bout, D. A. Utilizing Redox-Chemistry to Elucidate the Nature of Exciton Transitions in Supramolecular Dye Nanotubes. Nat. Chem. 2012, 4, 655-662.

(50) Günther, L. M.; Löhner, A.; Reiher, C.; Kunsel, T.; Jansen, T. L. C.; Tank, M.; Bryant, D. A.; Knoester, J.; Köhler, J. Structural Variations in Chlorosomes from Wild- Type and a bchQR Mutant of Chlorobaculum Tepidum Revealed by Single-Molecule Spectroscopy. J. Phys. Chem. B 2018, 122, 6712-6723.

(51) Eisfeld, A.; Briggs, J. S. The J- and H-bands of Organic Dye Aggregates. Chem. Phys. 2006, 324, 376-384.

(52) Even, J.; Carignano, M.; Katan, C. Molecular Disorder and Translation/Rotation Coupling in the Plastic Crystal Phase of Hybrid Perovskites. Nanoscale 2016, 8, 6222-6236.

(53) Frenkel, J. On the Transformation of Light into Heat in Solids. I. Phys. Rev. 1931, 37, 17-44.

(54) Jang, S. J.; Mennucci, B. Delocalized Excitons in Natural LightHarvesting Complexes. Rev. Mod. Phys. 2018, 90, No. 035003.

(55) Dostál, J.; Mančal, T.; Vacha, F.; Pšenčík, J.; Zigmantas, D. Unraveling the Nature of Coherent Beatings in Chlorosomes. J. Chem. Phys. 2014, 140, 115103.
(56) Jun, S.; Yang, C.; Isaji, M.; Tamiaki, H.; Kim, J.; Ihee, H. Coherent Oscillations in Chlorosome Elucidated by Two-Dimensional Electronic Spectroscopy. J. Phys. Chem. Lett. 2014, 5, 1386-1392.

(57) Rathbone, H. W.; Davis, J. A.; Michie, K. A.; Goodchild, S. C.; Robertson, N. O.; Curmi, P. M. Coherent Phenomena in Photosynthetic Light Harvesting: Part Two - Observations in Biological Systems. Biophys. Rev. 2018, 10, 1443-1463.

(58) Alia, A.; Buda, F.; de Groot, H. J. M.; Matysik, J. Solid-State NMR of Nanomachines Involved in Photosynthetic Energy Conversion. Annu. Rev. Biophys. 2013, 42, 675-699.

(59) Dama, J. F.; Sinitskiy, A. V.; McCullagh, M.; Weare, J.; Roux, B.; Dinner, A. R.; Voth, G. A. The Theory of Ultra-Coarse-Graining. 1. General Principles. J. Chem. Theory Comput. 2013, 9, 2466-2480.

(60) Tapavicza, E.; Bellchambers, G. D.; Vincent, J. C.; Furche, F. Ab Initio Non-Adiabatic Molecular Dynamics. Phys. Chem. Chem. Phys. 2013, 15, 18336-18348.

(61) Pšenčík, J.; Polívka, T.; Němec, P.; Dian, J.; Kudrna, J.; Malý, P.; Hála, J. Fast Energy Transfer and Exciton Dynamics in Chlorosomes of the Green Sulfur Bacterium Chlorobium tepidum. J. Phys. Chem. A 1998, 102, 4392-4398.

(62) Dostál, J.; Pšenčík, J.; Zigmantas, D. In Situ Mapping of the Energy Flow through the Entire Photosynthetic Apparatus. Nat. Chem. 2016, 8, 705-710. 